NBER WORKING PAPER SERIES

\title{
ON THE UNSTABLE RELATIONSHIP BETWEEN EXCHANGE RATES AND MACROECONOMIC FUNDAMENTALS
}

Philippe Bacchetta

Eric van Wincoop

Working Paper 15008

http://www.nber.org/papers/w15008

\author{
NATIONAL BUREAU OF ECONOMIC RESEARCH \\ 1050 Massachusetts Avenue \\ Cambridge, MA 02138
}

May 2009

We would like to thank Raf Wouters, Harald Uhlig, Luca Dedola and other seminar participants at Breugel, Tilburg, Bocconi, Dutch Central Bank, ECB, University of Lausanne and University of Zurich for useful comments and suggestions. We also thank Toni Beutler for able research assistance. We gratefully acknowledge financial support from the Bankard Fund for Political Economy, the National Science Foundation (grant SES-0649442) (van Wincoop), the National Centre of Competence in Research "Financial Valuation and Risk Management" (NCCR FINRISK), the Swiss Finance Institute and the Hong Kong Institute for Monetary Research (Bacchetta). The views expressed herein are those of the author(s) and do not necessarily reflect the views of the National Bureau of Economic Research.

NBER working papers are circulated for discussion and comment purposes. They have not been peerreviewed or been subject to the review by the NBER Board of Directors that accompanies official NBER publications.

(C) 2009 by Philippe Bacchetta and Eric van Wincoop. All rights reserved. Short sections of text, not to exceed two paragraphs, may be quoted without explicit permission provided that full credit, including (c) notice, is given to the source. 
On the Unstable Relationship between Exchange Rates and Macroeconomic Fundamentals Philippe Bacchetta and Eric van Wincoop

NBER Working Paper No. 15008

May 2009

JEL No. F31,F37,F41

\begin{abstract}
$\underline{\text { ABSTRACT }}$
It is well known from anecdotal, survey and econometric evidence that the relationship between the exchange rate and macro fundamentals is highly unstable. This could be explained when structural parameters are known and very volatile, neither of which seems plausible. Instead we argue that large and frequent variations in the relationship between the exchange rate and macro fundamentals naturally develop when structural parameters in the economy are unknown and change very slowly. We show that the reduced form relationship between exchange rates and fundamentals is driven not by the structural parameters themselves, but rather by expectations of these parameters. These expectations can be highly unstable as a result of perfectly rational "scapegoat" effects. This happens when parameters can potentially change much more in the long run than the short run. This generates substantial uncertainty about the level of parameters, even though monthly or annual changes are small. This mechanism can also be relevant in other contexts of forward looking variables and could explain the widespread evidence of parameter instability found in macroeconomic and financial data. Finally, we show that parameter instability has remarkably little effect on the volatility of exchange rates, the in-sample explanatory power of macro fundamentals and the ability to forecast out of sample.
\end{abstract}

\author{
Philippe Bacchetta \\ Faculty of Business and Economics \\ University of Lausanne \\ Extranef \\ CH-1015 Lausanne \\ Switzerland \\ philippe.bacchetta@unil.ch \\ Eric van Wincoop \\ Department of Economics \\ University of Virginia \\ P.O. Box 400182 \\ Charlottesville, VA 22904-4182 \\ and NBER \\ vanwincoop@virginia.edu
}




\section{Introduction}

"The dollar's resilience in the wake of recent dire US economic data has raised the prospect that the currency market may be experiencing one of its periodic changes in focus" (Financial Times, February 11, 2008)

"The dollar's latest stumble ... came despite optimistic economic data from the US. But analysts said the movement of the US currency was no longer driven by growth fundamentals. All the focus is on the deficit now..." (Financial Times, February 11,2003$)$

As reflected in these quotes, foreign exchange traders regularly change the weight they attach to different macro indicators. Cheung and Chinn (2001) have documented these changes through a survey of U.S. foreign exchange traders. Frequent changes in focus lead to an unstable relationship between exchange rates and macro fundamentals. Such parameter instability is confirmed in formal econometric evidence. Rossi (2005) conducts a battery of parameter instability tests and finds "overwhelming evidence of parameter instability". Sarno and Valente (2008) find that "(exchange rate) models that optimally use the information in the fundamentals change often and this implies frequent shifts in the parameters". Such instability has also been reported by Meese and Rogoff (1988) and Meese and Rogoff $(1983 \mathrm{a}, \mathrm{b})$ conjectured that it may explain the poor out-of-sample forecast ability of exchange rate models.

One way to explain the highly unstable relationship between exchange rates and macro fundamentals is to assume large and frequent changes in structural parameters that are known to all agents. This does not appear very plausible though as these parameters are not directly observed and hard to estimate. Moreover, many structural changes in the economy, such as those associated with technological and financial innovation and institutional reform, are gradual.

The main goal of this paper is to show that large and frequent variations in the relationship between the exchange rate and macro fundamentals can occur quite naturally even when structural parameters in the economy are unknown and change very slowly. We show that the relationship between a forward looking variable like the exchange rate and macro fundamentals is determined not by the 
structural parameters themselves, but rather by the expectations of these structural parameters. We show that these expectations can vary significantly over time, giving rise to a highly unstable reduced form relationship between exchange rates and fundamentals. This happens even though agents are perfectly rational Bayesian learners and changes in structural parameters are small and gradual.

While the focus of this paper is on exchange rates, our explanation for the unstable reduced-form relationship could apply similarly to other forward looking financial or macroeconomic variables. As first shown by Stock and Watson (1996), and since then by many others, the phenomenon of parameter instability in macroeconomic data is widespread. ${ }^{1}$ The same is the case for financial data. In a survey, Pastor and Veronesi (2009) point out that "parameter uncertainty is ubiquitous in finance" and "many facts that appear baffling at first sight seem less puzzling once we recognize that parameters are uncertain and subject to learning". ${ }^{2}$

The estimation mistakes that agents make when continuously updating their views on structural parameters are to a large extent a result of what we refer to as "scapegoat" effects. Some information about the nature of structural parameters can be derived by analyzing macroeconomic data and exchange rates. But these data are also driven by shocks to unobserved fundamentals. Such unobserved fundamentals can generate considerable confusion in the short to medium run. When the exchange rate fluctuates as a result of an unobserved macroeconomic shock, it can be optimal for agents to blame this on an observed macro fundamental by giving it more weight and therefore making it a "scapegoat". ${ }^{3}$ For example, when the

\footnotetext{
${ }^{1}$ Recent contributions include Boivin (2006), Canova (2005), Clarida, Gali and Gertler (2000), Cogley and Sargent (2005), Del Negro and Otrok (2007), Inoue and Rossi (2007), Primiceri (2005), Sims and Zha (2006) and Fernandez-Villaverde and Rubio-Ramirez (2007). There has also been great interest in the impact of parameter or model uncertainty on optimal monetary policy. See for example contributions by Hansen and Sargent (2008), Onatski and Williams (2003) or Levin et al. (2006).

${ }^{2}$ For example, Cogley (2005) and Piazzesi and Schneider (2007) introduce uncertainty about time-varying parameters to explain the term spread.

${ }^{3}$ In a previous short paper, Bacchetta and van Wincoop (2004), we developed the idea of such a scapegoat effect in the context of a simple static noisy rational expectations model in which some parameters are unknown. We showed that excessive weight could be given to a variable depending on the correlation between the noise shock and the fundamental shock. However, since that model is static it could not be used to address the unstable dynamic relationship between exchange rates and fundamentals and its implications. Apart from the dynamic setup, the model
} 
dollar depreciates it is natural to attribute it to a large current account deficit. This happens even when the depreciation is unrelated to the current account deficit.

There is significant potential for such scapegoat effects when the uncertainty about structural parameters is large. Two factors contribute to this. First, parameters can potentially change much more in the long-run than the shortrun. This generates substantial uncertainty about the level of parameters, even though monthly or annual changes are small. Second, agents cannot observe these structural parameters and obtain only very indirect information about their level through inference from the data.

In illustrating the importance of such scapegoat effects and their role in the unstable reduced form relationship between exchange rates and fundamentals, we slightly generalize the "canonical" exchange rate model. This is actually a broad class of exchange rate models that can be reduced to a single stochastic difference equation, which is derived from two equations: an interest rate parity equation and an equation that relates the interest differential to observed fundamentals. The latter can be obtained either from monetary policy specifications or money market equilibrium in a standard monetary model (see Engel and West, 2005, for examples). We generalize this by introducing time variation in the interest rate differential equation. While we illustrate the source of this time variation in the context of the standard monetary model, in general it can have many possible sources. Examples are changes in monetary policy parameters, changes in money demand parameters, or changes in the relationship between policy targets and observed fundamentals.

We calibrate the model to data for 5 industrialized countries, matching moments related to interest rates and exchange rates and the explanatory power of observed fundamentals. We consider a particular process for time-varying structural parameters that satisfies two features. First, changes in these parameters are small over short horizons of a month or a year. Second, changes in structural parameters gradually build over time, so that they can change substantially over long periods. These features are plausible when we think of long-term technological, institutional or cultural changes. Such a process generates large scapegoat in this paper also differs in that there is no private information as in Bacchetta and van Wincoop (2004). Scapegoat effects naturally develop as long as there is incomplete information about parameters; the information does not need to be private. 
effects as there is substantial uncertainty about the level of parameters even when month-to-month changes are small.

We do not estimate the process of structural parameters. That would be nearly impossible to do. First, the data can tell us very little about the exact nature of the process of time-varying parameters, even if there is clear evidence of parameter instability (e.g., see Elliott and Timmermann, 2008, for a discussion). Second, even when a particular process is assumed, its parameters are notoriously hard to estimate with any precision. While we focus on a specific process in the benchmark analysis, we examine the robustness of our results to a wide range of alternative processes.

We also use the model to evaluate the impact of reduced-form parameter instability on the ability to forecast out of sample. It is exactly in this context that the issue of parameter instability has most frequently been discussed in the exchange rate literature. ${ }^{4}$ Meese and Rogoff (1983) first showed that models do not outperform the random walk in forecasting future exchange rates, even when the actual future macro fundamentals are used to forecast. Their results have largely held up since then, even with a lot more data available. ${ }^{5}$ Meese and Rogoff (1983a,b) conjectured that time-varying parameters may be responsible for this poor out-of-sample performance as estimated parameters can be quite different from parameters over the forecast horizon. Nonetheless we obtain the surprising result that time-varying parameters have very little impact on the out-of-sample forecasting performance relative to the random walk. We explain what accounts for this surprising result in conjunction with a companion paper, Bacchetta, van Wincoop, and Beutler (2009), which considers the impact of exogenous reducedform parameter instability on the out-of-sample performance of the model relative to the random walk.

The next section presents the model. It also discusses the signal extraction method used to solve the model and the implications for the relationship between exchange rates and fundamentals. Section 3 calibrates the model based on data on interest rates and exchange rates and presents numerical results for the relationship between exchange rates and fundamentals based on simulations. Section 4 uses the

\footnotetext{
${ }^{4}$ See Wolff (1987), Schinasi and Swamy (1989), Rossi (2005), Sarno and Valente (2008) and Meese and Rogoff (1988).

${ }^{5}$ See for example Cheung, Chinn and Pascual (2005) and Rogoff and Stavrakeva (2008).
} 
model to analyze the impact of the unstable relationship between exchange rates and fundamentals on the ability to forecast out of sample. Section 5 concludes.

\section{A Model with Unknown Parameters}

We first describe the model when parameters are constant and known. Then we introduce unknown and time-varying coefficients and examine how the impact of fundamentals on the exchange rate is affected. For that purpose, we need to derive how expectations about parameters are formed. We show that this process leads to an unstable relationship between fundamentals and exchange rates. The final subsection provides intuition on the mechanism leading to this instability.

\subsection{Basic Framework with Constant Parameters}

We consider the class of fundamental-based exchange rate models that can be reduced to a single stochastic difference equation. The equilibrium value of the exchange rate in these models depends on the present value of expected future fundamentals. We start with the usual case of constant and known parameters. We follow Engel and West (2005) and slightly rewrite their equation (1):

$$
s_{t}=(1-\lambda)\left[F_{t}+b_{t}+\sum_{j=1}^{\infty} \lambda^{j} E_{t}\left(F_{t+j}+b_{t+j}\right)\right]-\lambda\left[\phi_{t}+\sum_{j=1}^{\infty} \lambda^{i} E_{t} \phi_{t+j}\right]
$$

where $s_{t}$ is the log nominal exchange rate (domestic per foreign currency), $E_{t}$ is the expectation of the representative investor, $\phi_{t}$ is the risk premium and $0<\lambda<1$. We denote by $F_{t}$ a linear combination of observed macro fundamentals: $F_{t}=\mathbf{f}_{t}^{\prime} \boldsymbol{\beta}$ where $\mathbf{f}_{t}=\left(f_{1 t}, f_{2 t}, \ldots, f_{N t}\right)^{\prime}$ is the vector of $N$ observed macroeconomic fundamentals and $\boldsymbol{\beta}=\left(\beta_{1}, \beta_{2}, \ldots, \beta_{N}\right)^{\prime}$ is the vector of associated parameters. Finally, $b_{t}$ represents unobserved macro fundamentals.

Engel and West (2005) present several models that lead to this equation. ${ }^{6}$ For illustrative purposes we focus on the familiar flexible-price monetary model. A two-country model can be described by the standard four equations:

$$
E_{t} s_{t+1}-s_{t}=i_{t}-i_{t}^{*}+\phi_{t}
$$

\footnotetext{
${ }^{6}$ See also Nason and Rogers (2008) who derive this equation from a DSGE model.
} 


$$
\begin{aligned}
s_{t} & =p_{t}-p_{t}^{*} \\
\mu m_{t} & =p_{t}-\alpha i_{t}+\gamma^{\prime} \mathbf{z}_{t}+\nu_{t} \\
\mu m_{t}^{*} & =p_{t}^{*}-\alpha i_{t}^{*}+\gamma^{\prime} \mathbf{z}_{t}^{*}+\nu_{t}^{*}
\end{aligned}
$$

As usual, $i_{t}$ and $i_{t}^{*}$ represent the domestic and foreign nominal one-period interest rates, $p_{t}$ and $p_{t}^{*}$ are the domestic and foreign log prices, and $m_{t}$ and $m_{t}^{*}$ are the log nominal money supplies. We denote by $\mathbf{z}_{t}$ and $\mathbf{z}_{t}^{*}$ the vectors of other observed fundamentals affecting money demand. Unobserved velocity shocks are denoted $\nu_{t}$ and $\nu_{t}^{*}$. The parameter $\mu$ is usually set at 1 , but does not need to be 1 when the vector $\mathbf{z}_{t}$ includes nominal variables as well. ${ }^{7}$

By combining equations (3), (4), and (5), we find:

$$
i_{t}-i_{t}^{*}=\frac{1}{\alpha} s_{t}-\frac{1}{\alpha}\left[\mu\left(m_{t}-m_{t}^{*}\right)-\gamma^{\prime}\left(\mathbf{z}_{t}-\mathbf{z}_{t}^{*}\right)\right]+\frac{1}{\alpha}\left(\nu_{t}-\nu_{t}^{*}\right)
$$

This equation can be rewritten in a more compact form as:

$$
i_{t}-i_{t}^{*}=\frac{1}{\alpha} s_{t}-\frac{1}{\alpha}\left(F_{t}+b_{t}\right)
$$

where $b_{t}=-\left(\nu_{t}-\nu_{t}^{*}\right)$ is an unobserved fundamental and $F_{t}=\mathbf{f}_{t}^{\prime} \boldsymbol{\beta}=\mu\left(m_{t}-m_{t}^{*}\right)-$ $\gamma^{\prime}\left(\mathbf{z}_{t}-\mathbf{z}_{t}^{*}\right)$ is a linear combination of observed fundamentals. Combining equations (2) and (7), integrating forward and assuming no bubble gives equation (1), where $\lambda=\alpha /(1+\alpha)$.

Since $s_{t}$ and fundamentals are typically non-stationary in the data, it is usual to consider first differences. As an illustration, consider the special case without a risk premium and where $b_{t}$ and $\Delta \mathbf{f}_{t}$ are iid. More precisely, assume that: i) $\phi_{t}=0$, $\forall t$; ii) $b_{t}=\varepsilon_{t}^{b}$ with $\varepsilon_{t}^{b} \sim N\left(0, \sigma_{b}^{2}\right)$; iii) $\Delta f_{n t}=\varepsilon_{n t}^{f}$ with $\varepsilon_{n t}^{f} \sim N\left(0, \sigma_{f}^{2}\right)$. In this case, we have:

$$
\Delta s_{t}=\Delta \mathbf{f}_{t}^{\prime} \boldsymbol{\beta}+(1-\lambda) \Delta b_{t}
$$

The impact of a change in fundamental $f_{n t}$ on the exchange rate is simply given by $\beta_{n}: \partial \Delta s_{t} / \partial \Delta f_{n t}=\beta_{n}$.

\footnotetext{
${ }^{7}$ Examples of nominal variables in $\mathbf{z}_{t}$ include lagged money demand, lagged prices or nominal financial wealth. Introducing $\mu$ gives us a parameter multiplying the money supply fundamental, just like $\gamma$ is a vector of parameters multiplying the other observed fundamentals $\mathbf{z}_{t}$. But it is not critical to the analysis in any substantial way.
} 


\subsection{Time-varying and Unknown Parameters}

We now depart from the standard model by assuming that the vector of coefficients $\mu$ and $\gamma$ varies over time and is not known by investors. ${ }^{8}$ However, simply replacing these constant parameters with a vector of time-varying parameters raises a technical problem that arises under any stationary process for the time-varying parameters. To see this, add a time-subscript to the parameters $\mu$ and $\gamma$ and then take the first-difference of (4):

$$
\mu_{t} \Delta m_{t}=\Delta p_{t}-\alpha \Delta i_{t}+\gamma_{t}^{\prime} \Delta \mathbf{z}_{t}+\Delta \nu_{t}+\Delta \gamma_{t}^{\prime} \mathbf{z}_{t-1}-\Delta \mu_{t} m_{t-1}
$$

In the data $\Delta m_{t}, \Delta p_{t}$ and $\Delta i_{t}$ are stationary. But this is inconsistent with nonstationarity in the last two terms. This non-stationarity arises because parameter changes are multiplied with the level of fundamentals, which can be unbounded due to non-stationarity. Both $m_{t}$ and elements of $\mathbf{z}_{t}$ are non-stationary in the data.

To solve this issue, we add the time subscript to the parameters $\mu$ and $\gamma$ after taking the first difference of (4):

$$
\mu_{t} \Delta m_{t}=\Delta p_{t}-\alpha \Delta i_{t}+\gamma_{t}^{\prime} \Delta \mathbf{z}_{t}+\Delta \nu_{t}
$$

This avoids the last two terms on the right hand side of (9) that are non-stationary. In level terms we can write this specification as

$$
\begin{aligned}
\tilde{m}_{t} & =p_{t}-\alpha i_{t}+Z_{t}+\nu_{t} \\
Z_{t} & =Z_{t-1}+\gamma_{t}^{\prime} \Delta \mathbf{z}_{t} \\
\tilde{m}_{t} & =\tilde{m}_{t-1}+\mu_{t} \Delta m_{t}
\end{aligned}
$$

Together with an analogous specification for money demand in the other country, and defining $F_{t}=\left(\tilde{m}_{t}-\tilde{m}_{t}^{*}\right)-\left(Z_{t}-Z_{t}^{*}\right)$, the solution for the interest differential remains the same as in (7). This again yields the present value equation (1) when combined with (2). This specification for $F_{t}$ implies that

$$
\Delta F_{t}=\Delta \mathbf{f}_{t}^{\prime} \boldsymbol{\beta}_{t}
$$

where

$$
\mathbf{f}_{t}=\left(\begin{array}{c}
m_{t} \\
\mathbf{z}_{t}
\end{array}\right) \quad ; \quad \boldsymbol{\beta}_{t}=\left(\begin{array}{c}
\mu_{t} \\
\gamma_{t}
\end{array}\right)
$$

\footnotetext{
${ }^{8}$ We assume, mainly for convenience, that the interest semi-elasticity $\alpha$ is known and constant.
} 
With a total of $N$ fundamentals we will also write $\boldsymbol{\beta}_{t}=\left(\beta_{1 t}, \beta_{2 t}, \ldots, \beta_{N t}\right)^{\prime}$.

We therefore replace the specification $\Delta F_{t}=\Delta \mathbf{f}_{t}^{\prime} \boldsymbol{\beta}$ for constant parameters with the specification $\Delta F_{t}=\Delta \mathbf{f}_{t}^{\prime} \boldsymbol{\beta}_{t}$ for time-varying parameters. While for illustrative purposes we have motivated this in the context of the familiar flexible price monetary model, it can also be obtained from other models that lead to the present value equation (1). One example is to replace the money market equilibrium by an interest rate rule that depends on a number of observed fundamentals. Timevarying parameters are then associated with time variation in the monetary policy parameters. Another possibility is that these monetary policy parameters are constant but the (possibly unknown) policy targets have a time-varying relationship to the observed fundamentals. The exact source of the time-varying parameters is not critical to the qualitative findings of the paper.

The major difference with the standard framework is that $F_{t}$ is not directly observable. Investors need to estimate current and future $\boldsymbol{\beta}_{t}$. They have two sources of information regarding $\boldsymbol{\beta}_{t}$. First, they know the process of $\boldsymbol{\beta}_{t}$, which we will specify below. Second, by observing the exchange rate and the interest rate differential, they know $F_{t}+b_{t}$ from (7). We describe below how investors combine optimally these two sources of information to form expectations about $\boldsymbol{\beta}_{t}$.

The signal $F_{t}+b_{t}$ provides information about the parameters, but is also a source of estimation errors. Consider for example the expectation of parameter $\beta_{n t}$ for fundamental $n$. While $\beta_{n t}$ affects $F_{t}+b_{t}$, the latter is also affected by $b_{t}$, all current and past fundamentals and all current and past parameters. Therefore, to the extent that $F_{t}+b_{t}$ is used as a source of information about $\beta_{n t}$, its expectation can change without any change in $\beta_{n t}$ itself. We will see that it is this rational confusion that is the key driver behind the unstable relationship between exchange rates and observed fundamentals.

\subsection{Exchange Rates and Fundamentals}

For convenience, in the remainder of this section we consider the special case without a risk premium and where $b_{t}$ and $\Delta \mathbf{f}_{t}$ are iid, as described above. A more general specification will be considered in the numerical analysis in the next section. We maintain the assumption throughout the paper that shocks to $f_{n t}, b_{t}$ and parameters are uncorrelated with each other. 
Under these assumptions, $(1-\lambda) \sum_{j=1}^{\infty} \lambda^{j} E_{t} F_{t+i}=\lambda E_{t} F_{t}$ because $E_{t} F_{t+i}=$ $E_{t} F_{t}$. The first difference of the present value equation (1) then becomes:

$$
\Delta s_{t}=(1-\lambda) \Delta F_{t}+(1-\lambda) \Delta b_{t}+\lambda\left(E_{t} F_{t}-E_{t-1} F_{t-1}\right)
$$

If the parameters $\boldsymbol{\beta}_{t}$ were known, then $F_{t}$ is known as well at time $t$ and (16) becomes

$$
\Delta s_{t}=\Delta \mathbf{f}_{t}^{\prime} \boldsymbol{\beta}_{t}+(1-\lambda) \Delta b_{t}
$$

This generalizes (8) by replacing the constant vector of parameters $\boldsymbol{\beta}$ that multiplies the fundamentals $\Delta \mathbf{f}_{t}^{\prime}$ by the vector of time-varying parameters $\boldsymbol{\beta}_{t}$. When the parameters $\beta_{n t}$ are not only known, but also very volatile, it could explain the unstable relationship between exchange rates and fundamentals.

However, in reality the time-varying parameters are unknown. In that case the last term $E_{t} F_{t}-E_{t-1} F_{t-1}$ in (16) is a complex expression that depends on expectations of parameters. In order to avoid the technical problem of computing expectations of parameter innovations going back to the infinite past, we assume that parameters are known after $T$ periods. Therefore the total number of unknown parameter innovations is $N T$, which is finite. In practice we will set $T$ very large. In that case, we can write (16) as:

$$
\Delta s_{t}=\Delta \mathbf{f}_{t}^{\prime}\left((1-\lambda) \boldsymbol{\beta}_{t}+\lambda E_{t} \boldsymbol{\beta}_{t}\right)+(1-\lambda) \Delta b_{t}+\lambda \sum_{i=1}^{T} \Delta \mathbf{f}_{t-i}^{\prime}\left(E_{t} \boldsymbol{\beta}_{t-i}-E_{t-1} \boldsymbol{\beta}_{t-i}\right)
$$

As can be seen from the first term in (18), $\Delta \mathbf{f}_{t}$ is now multiplied by a weighted average of actual and expected parameter values. Since the discount rate $\lambda$ tends to be close to 1 (see Engel and West, 2005), almost all of the weight is on the expected value of parameters rather than the actual level of parameters. The reason is that the exchange rate is forward looking and depends on expectations of future fundamentals. In this particular example, where fundamentals follow a random walk, expected future levels of $F$ are equal to the expected level of $F$ today, which depends on the expectation of the current set of parameters $\boldsymbol{\beta}_{t}$. More generally, if changes in fundamentals are not iid, $\Delta s_{t}$ also depends on expectations about future values of the parameters. The general setup is discussed in Appendix A.

In the last term of (18) we see that $\Delta s_{t}$ also depends on the change in expectations $E_{t} \boldsymbol{\beta}_{t-i}-E_{t-1} \boldsymbol{\beta}_{t-i}$ of past parameters, interacted with changes in past 
fundamentals. Intuitively, since $F_{t}=\sum_{i=0}^{\infty} \Delta \mathbf{f}_{t-i}^{\prime} \boldsymbol{\beta}_{t-i}$, changes in the expectation of past parameters lead to a change in the expectation of $F_{t}$ and therefore the exchange rate. We will show that changes in current fundamentals lead to changes in the expectation of both current and past parameters. This is therefore an additional channel through which changes in current fundamentals affect the exchange rate.

To examine the impact of fundamentals on the exchange rate, we simply consider the derivative of the exchange rate with respect to current fundamentals:

$$
\frac{\partial \Delta s_{t}}{\partial \Delta f_{n t}}=(1-\lambda) \beta_{n t}+\lambda E_{t} \beta_{n t}+\lambda \sum_{i=0}^{T} \Delta \mathbf{f}_{t-i}^{\prime} \frac{\partial E_{t} \boldsymbol{\beta}_{t-i}}{\partial \Delta f_{n t}}
$$

The rest of this section analyzes in more detail the last two elements on the righthand side of (19).

\subsection{Expectation of Parameters}

In order to determine the impact of fundamentals on the exchange rate, we need to determine the expectation of current and past parameters. We do this by first assuming a process for structural parameters and then solving a signal extraction problem.

We consider the case where a structural parameter $\beta_{n t}$ depends on a finite number $T$ of past innovations:

$$
\beta_{n t}=\beta_{n}+\sum_{i=1}^{T} \theta_{n i} \varepsilon_{n, t-i+1}
$$

where $\varepsilon_{n t} \sim N\left(0, \sigma_{\beta}^{2}\right)$. In this section we consider a rather general process characterized by the parameters $\theta_{n i}$. In the next section we will pick a particular process for the numerical analysis that satisfies the criteria discussed in the Introduction.

As discussed in section 2.3, we assume that parameter innovations at dates $t-T$ and earlier are known at date $t$ in order avoid an infinite number of unknown parameter innovations about which expectations need to be formed. In addition (20) assumes that parameter innovations at $t-T$ and earlier do not affect parameters at time $t$. This is a different assumption, which we make to assure stationarity of the structural parameters. In practice the impact of these assumptions is minimized by setting $T$ very high in the numerical analysis. In addition we will consider an 
alternative process in section 3.3 where parameters depend on an infinite number of lagged innovations.

In vector notation (20) can be written as

$$
\boldsymbol{\beta}_{t}=\boldsymbol{\beta}+\Theta \boldsymbol{\xi}_{t}
$$

where $\boldsymbol{\beta}=\left(\beta_{1}, \beta_{2}, \ldots, \beta_{N}\right)^{\prime}$ is a $N$-vector of constants; $\boldsymbol{\xi}_{t}$ is a $N T$ vector that stacks all the vectors $\boldsymbol{\xi}_{n t}=\left(\epsilon_{n t}, \ldots, \epsilon_{n, t-T+1}\right)^{\prime}$; and $\Theta$ is a $N \times N T$ matrix with $\Theta[n, T(n-$ $1)+1: T n]=\boldsymbol{\theta}_{n}^{\prime}=\left(\theta_{n 1}, \theta_{n 2}, \ldots, \theta_{n T}\right)$ and zeros otherwise.

In order to form expectations about current and past values of $\boldsymbol{\beta}_{t}$ we need to compute expectations about the vector $\boldsymbol{\xi}_{t}$ of current and past parameter innovations. Since the problem is linear and all the shocks are normal, we can use standard signal extraction techniques. Leaving some of the details to Appendix B, we sketch how this is done. We start from the knowledge that the unconditional distribution of $\boldsymbol{\xi}_{t}$ is normal with mean zero and variance $\sigma_{\beta}^{2} \mathbf{I}_{N T}$, where $\mathbf{I}_{N T}$ is an identity matrix of size $N T$. We combine this with knowledge of $d_{t}=F_{t}+b_{t}$ over the past $T$ periods. Defining $\mathbf{Y}_{t}=\left(d_{t}^{*}, . ., d_{t-T+1}^{*}\right)^{\prime}$, where $d_{t}^{*}$ subtracts the known components from $d_{t}$, we have

$$
\mathbf{Y}_{t}=\mathbf{H}_{t}^{\prime} \boldsymbol{\omega}_{t}
$$

where $\boldsymbol{\omega}_{t}^{\prime}=\left(\boldsymbol{\xi}_{t}^{\prime}, \varepsilon_{t}^{b}, \varepsilon_{t-1}^{b}, \ldots, \varepsilon_{t-T+1}^{b}\right)$ and $\mathbf{H}_{t}$ is a matrix that depends on current and lagged changes in observed fundamentals: $\Delta \mathbf{f}_{t-i}$ for $0 \leq i \leq T$. The precise form of $\mathbf{H}_{t}$ can be found in Appendix B.

The unconditional distribution of $\boldsymbol{\omega}_{t}$ is normal with mean zero and variance

$$
\tilde{\mathbf{P}}=\left(\begin{array}{cc}
\sigma_{\beta}^{2} \mathbf{I}_{N T} & \mathbf{0} \\
\mathbf{0} & \sigma_{b}^{2} \mathbf{I}_{T}
\end{array}\right)
$$

Combining this with $(22)$, standard signal extraction ${ }^{9}$ implies that the conditional distribution of $\boldsymbol{\omega}_{t}$ is normal with mean

$$
\begin{aligned}
& E_{t} \boldsymbol{\omega}_{t}=\mathbf{M}_{t} \mathbf{Y}_{t} \\
& \mathbf{M}_{t}=\tilde{\mathbf{P}} \mathbf{H}_{t}\left[\mathbf{H}_{t}^{\prime} \tilde{\mathbf{P}} \mathbf{H}_{t}\right]^{-1}
\end{aligned}
$$

and variance

$$
\mathbf{P}_{t}=\tilde{\mathbf{P}}-\mathbf{M}_{t} \mathbf{H}_{t}^{\prime} \tilde{\mathbf{P}}
$$

\footnotetext{
${ }^{9}$ See for example Townsend(1983, p.556).
} 
Therefore

$$
E_{t} \boldsymbol{\omega}_{t}=\mathbf{C}_{t} \boldsymbol{\omega}_{t}
$$

where $\mathbf{C}_{t}=\mathbf{M}_{t} \mathbf{H}_{t}^{\prime}$. Together with knowledge of parameter innovations of at least $T$ periods ago, (25) gives expressions for $E_{t} \boldsymbol{\xi}_{t-i}$, for $i=0,1, \ldots, T-1$. We use this to compute $E_{t} \boldsymbol{\beta}_{t-i}$ from (21).

We then have

$$
E_{t} \boldsymbol{\beta}_{t-i}=\hat{\boldsymbol{\beta}}_{t-i}+\boldsymbol{\Omega}_{t i} \boldsymbol{\omega}_{t}
$$

Here $\hat{\boldsymbol{\beta}}_{t-i}$ is equal to $\boldsymbol{\beta}$ plus (for $i>0$ ) a vector that depends on parameter innovations of at least $T$ periods ago that are known at time $t$. The matrix $\boldsymbol{\Omega}_{t i}$ is equal to $\Theta \widetilde{\mathbf{I}}_{i} \mathbf{C}_{t}$, where $\widetilde{\mathbf{I}}_{i}$ is a matrix of zeros and ones that maps $\boldsymbol{\omega}_{t}$ into the unknown elements of $\boldsymbol{\xi}_{t-i}$.

There are two important features to notice from (26). First, $E_{t} \boldsymbol{\beta}_{t-i}$ is determined by a combination of shocks contained in $\boldsymbol{\omega}_{t}$. Thus, the expectation of a specific parameter $\beta_{n t-i}$ depends on its own innovations, but also on current and past innovations to the noise vector $\mathbf{b}_{t}$ and to all other parameters. Second, $\boldsymbol{\Omega}_{t i}$ depends on current and past $\Delta \mathbf{f}_{t}$ so that shocks to fundamentals affect parameter expectations. ${ }^{10}$

As we will see, the expectation of $\beta_{n t}$ can change significantly over a relatively short period even when the actual structural parameters change very slowly. What matters is not the monthly (or even annual) fluctuations in structural parameters but rather their potential to fluctuate over a very long period of time (decades or longer). The unconditional standard deviation of the parameters then becomes large even though changes from period to period are small. A large unconditional standard deviation of structural parameters, together with the difficulty in learning about their level, may imply large and frequent changes in expectations about these parameters. This allows expectations to become significantly disconnected from the true value of the parameters.

\footnotetext{
${ }^{10}$ Current and past $\Delta \mathbf{f}_{t}$ enter $\mathbf{H}_{t}$, which affects $\mathbf{M}_{t}, \mathbf{C}_{t}$, and therefore $\boldsymbol{\Omega}_{t i}$.
} 


\subsection{Derivative of Exchange Rate with respect to Funda- mentals: Intuition}

After substituting the solution for the expected parameters into (19), we have an expression for the derivative of the exchange rate with respect to fundamentals as a function of all the underlying shocks in the model: shocks to fundamentals, $\varepsilon_{n t}^{f}$, shocks to parameters, $\varepsilon_{n t}$, and shocks to unobservables, $\varepsilon_{t}^{b}$. We can solve the model numerically to show how the derivative evolves over time. However, it is hard to get much intuition out of the algebraic expression. It is highly nonlinear in the shocks, which enter through large matrices and their inverse. To provide some intuition, especially regarding the scapegoat effect, in this section we discuss the first and second-order components of the derivative. ${ }^{11}$ This allows us to show that the impact of the actual structural parameters is significantly dampened relative to the case where the parameters are known, while shocks to the unobserved fundamentals $b_{t}$ have a significant impact.

For convenience we repeat expression (19) for the derivative of the exchange rate with respect to fundamentals:

$$
\frac{\partial \Delta s_{t}}{\partial \Delta f_{n t}}=(1-\lambda) \beta_{n t}+\lambda E_{t} \beta_{n t}+\lambda \sum_{i=0}^{T} \Delta \mathbf{f}_{t-i}^{\prime} \frac{\partial E_{t} \boldsymbol{\beta}_{t-i}}{\partial \Delta f_{n t}}
$$

A Technical Appendix that is available on request computes the various order components of this expression. The first term on the right hand side, $(1-\lambda) \beta_{n t}$, only has zero and first-order components. The second term, $\lambda E_{t} \beta_{n t}$, has components of all orders. The last term has second and higher-order components. Since third and higher-order components tend to be small, we only discuss the solution up to the second order. We will assume that all $N$ parameters are drawn from the same process, so that $\beta_{n}=\beta$ and $\theta_{n i}=\theta_{i}$ for all $n$.

The zero-order component of the derivative (27) is simply $\beta$, the steady state

\footnotetext{
${ }^{11}$ Any variable can be written as the sum of its components of all orders. For example, for a variable $x_{t}$ we have $x_{t}=x(0)+x_{t}(1)+x_{t}(2)+\ldots$ The zero-order component of a variable, $x(0)$, is its value when the standard deviation of shocks in the model approaches zero. The first-order component, $x_{t}(1)$, is proportional to the shocks. The second-order component, $x_{t}(2)$, is proportional to the product of two shocks (or the same shock squared). Notice that we only compute these order components for the purpose of intuition. The simulations reported below are based on the exact expressions.
} 
value of the parameter. The first-order component is

$$
\frac{\partial \Delta s_{t}}{\partial \Delta f_{n t}}(1)=(1-\lambda)\left(\beta_{n t}-\beta\right)
$$

In comparison to the case where parameters are time varying but known, where the derivative is $\beta_{n t}$, the impact of the actual structural parameter is now dampened by $1-\lambda$. Since in practice $\lambda$ is close to 1 , this means that the actual structural parameters have very little effect on the derivative. In order to understand this, consider the first two terms on the right hand side of the derivative expression (27), $(1-\lambda) \beta_{n t}+\lambda E_{t} \beta_{n t}$. Most of the weight $(\lambda)$ is on the expectation of $\beta_{n t}$. But to the first-order parameter expectations are unaffected by actual parameter innovations.

To illustrate that parameter innovations have no first-order effect on the expectation of parameters, consider the simple case where $T=N=1$. In this case, only the most recent parameter innovation $\varepsilon_{t}$ is unknown. Apart from knowing the unconditional distribution of $\varepsilon_{t}$, we have one other signal: $F_{t}+b_{t}$, for which the only unknown component in this case is simply $\varepsilon_{t} \Delta f_{t}+\varepsilon_{t}^{b}$. This signal is not very informative about $\varepsilon_{t}$ as it is multiplied by $\Delta f_{t}$, a first-order shock rather than a zero-order constant. In this simple case the expectation of the parameter innovation is

$$
E_{t} \varepsilon_{t}=\frac{\sigma_{\beta}^{2}}{\left(\Delta f_{t}\right)^{2} \sigma_{\beta}^{2}+\sigma_{b}^{2}}\left(\left(\Delta f_{t}\right)^{2} \varepsilon_{t}+\Delta f_{t} \varepsilon_{t}^{b}\right)
$$

It is easily verified that the term involving the parameter innovation $\varepsilon_{t}$ has firstand second-order components equal to zero. Only the third-order component is different from zero: $\left(\sigma_{\beta}^{2} / \sigma_{b}^{2}\right)\left(\Delta f_{t}\right)^{2} \varepsilon_{t}$. This holds more generally when $N$ and $T$ differ from 1: only third and higher order components of $E_{t} \beta_{n t}$ depend on parameter innovations.

The second-order component of the derivative is

$$
\frac{\partial \Delta s_{t}}{\partial \Delta f_{n t}}(2)=\frac{\sigma_{\beta}^{2}}{\sigma_{b}^{2}} \lambda \sum_{i=0}^{T-1} \zeta_{t, i} \varepsilon_{t-i}^{b}+\frac{\sigma_{\beta}^{2}}{\sigma_{b}^{2}} \lambda \vartheta_{t} \varepsilon_{t}^{b}
$$

where:

$$
\begin{aligned}
\zeta_{t, i} & =\sum_{k=i}^{T-1} \sum_{j=1}^{T-k} \theta_{j} \theta_{j+k} \delta_{i k} \Delta f_{n, t-k} \\
\vartheta_{t} & =\sum_{i=0}^{T}\left(\sum_{j=1}^{T-i} \theta_{j} \theta_{j+i}\right) \Delta f_{n, t-i}
\end{aligned}
$$


and where $\delta_{i k}$ is 1 for $k=i$ and $1-\rho_{b}$ for $k>i$.

The first term on the right hand side of (30) is equal to the second-order component of $\lambda E_{t} \beta_{n t}$. Two surprising aspects stand out. First, the noise shocks (shocks to unobserved fundamentals) have a bigger impact on the expectation of the structural parameters than structural parameter innovations themselves. The impact of noise shocks on $E_{t} \beta_{n t}$ is second-order, while our previous discussion showed that the impact of actual parameter innovations is third-order. ${ }^{12}$ Second, the second-order component of $\lambda E_{t} \beta_{n t}$ involves a product of noise innovations (current and past) and innovations in the observed fundamentals (current and past). This reflects what we call a scapegoat effect.

In order to understand the scapegoat effect, imagine that innovations in unobserved fundamentals have been high, so that $b_{t}$ is high. But since agents cannot observe $b_{t}$, they only see that $F_{t}+b_{t}$ is high. They will then increase their expectation of parameters whose fundamental innovations have been large (positive). After all, a large fundamental combined with a high value of its parameter can also explain the large value of $F_{t}+b_{t}$. Such a variable then becomes a scapegoat. This is a result of fully rational confusion as agents form expectations based on all available information. It explains why the expectation of a parameter depends positively on the product of innovations in the associated fundamental and unobservable noise innovations.

Next consider the second term on the right hand side of (30). This term also reflects a scapegoat effect. It again involves the product of fundamental innovations with a noise innovation. But in this case it is only the current noise innovation $\varepsilon_{t}^{b}$ that enters. Therefore this term is uncorrelated across time. The absence of any persistence implies that this component leads to very high frequency fluctuations in the derivative.

This term captures the second-order component of the last term in the derivative (27). The impact of a fundamental innovation $\Delta f_{n t}$ on the exchange rate depends not only on the expectation of $\beta_{n t}$ that multiplies the fundamental innovation. It also depends on how the change in the fundamental leads to changes in the expectation of current and past parameters, as seen in the last term of (27). A change in the current fundamental affects current and past parameter expectations

\footnotetext{
${ }^{12}$ The second-order impact of noise innovations on the expectation of parameters is also seen in (29), where the term involving the unobservable innovation $\varepsilon_{t}^{b}$ is of second order: $\left(\sigma_{\beta}^{2} / \sigma_{b}^{2}\right) \Delta f_{t} \varepsilon_{t}^{b}$.
} 
only to the extent that it becomes a scapegoat in the face of a current noise shock $\epsilon_{t}^{b}$. To see this last point, one can alternatively write the signals $F_{t-i}+b_{t-i}$ for $i=0, . ., T-1$ as $\left(1-\rho_{b} L\right)\left(F_{t-i}+b_{t-i}\right)=F_{t-i}-\rho_{b} F_{t-i-1}+\varepsilon_{t}^{b}$. The current fundamental innovation $\Delta f_{n t}$ only enters in the most recent signal $(i=0)$, in which only the most recent noise innovation enters.

One other aspect of the second-order expression (30) should be emphasized. The parameters $\zeta_{t, i}$ and $\vartheta_{t}$ depend on the products $\theta_{j} \theta_{j+k}$ of coefficients of the process of structural parameters. Coefficients $\theta_{j}$ for $j>1$ capture the persistence of the impact of parameter innovations on the level $\beta_{n t}$ of structural parameters. Clearly, the more persistence, the larger $\zeta_{t, i}$ and $\vartheta_{t}$ and therefore the bigger the scapegoat effects. This reflects the fact that when parameter innovations have long-lasting effects on the structural parameters, there is significant uncertainty about the level of the structural parameters, which leaves plenty of room for the scapegoat mechanism to operate. This is especially the case when $\theta_{j}$ rises with $j$, which implies a gradual change in parameters in response to an innovation, so that parameters can change much more in the long run than the short run.

While we do not report the third-order component, it is worth pointing out that it captures another type of rational confusion. Instead of confusing unobserved parameter shocks with unobserved fundamental shocks, agents may also confuse the unobserved innovations in one parameter with unobserved innovations in another parameter. This is reflected in the third-order component, which is a complicated expression that multiplies current and past parameter innovations (including those associated with other parameters) with the product of fundamental innovations (current and past).

In order to illustrate these points and show the magnitude of the scapegoat effect, we now turn to a calibration of the model that is grounded in monthly data of exchange rates and interest rates.

\section{Numerical Analysis}

\subsection{Calibration}

We calibrate the model to data for exchange rates, interest rates and observed fundamentals. A description of the data can be found in Appendix C. In the 
previous section, we considered a special case with no risk-premium shocks and where both $b_{t}$ and $\Delta \mathbf{f}_{n t}$ are iid. For calibration purposes we now turn to a somewhat more general form of the model.

First, we assume that $b_{t}$ and $\Delta f_{n t}$ follow $\mathrm{AR}(1)$ processes:

$$
\begin{aligned}
\Delta f_{n t} & =\rho_{f} \Delta f_{n, t-1}+\varepsilon_{t}^{f} \\
b_{t} & =\rho_{b} b_{t-1}+\varepsilon_{t}^{b}
\end{aligned}
$$

Second, in order to match observed exchange rate volatility we allow for a timevarying risk premium. Let $v_{t}$ be the present discounted value of the risk premium:

$$
v_{t}=\sum_{k=0}^{\infty} \lambda^{k} E_{t} \phi_{t+k}
$$

To match the observed volatility and autocorrelation of $\Delta s_{t}$, we assume that $v_{t}$ follows the process

$$
v_{t+1}-v_{t}=\psi_{1}\left(v_{t}-v_{t-1}\right)-\psi_{2} v_{t}+\varepsilon_{t+1}^{v}
$$

where $\varepsilon_{t+1}^{v} \sim N\left(0, \sigma_{v}^{2}\right){ }^{13}$

The process for the structural parameters is determined by the values of the parameters $\theta_{\text {in }}$ in equation (20). We assume that the parameters associated with all observed fundamentals are the same, so that $\beta_{n}=\beta$ and $\theta_{i n}=\theta_{i}$ for all $n$. As discussed in the introduction, we consider structural parameters that exhibit two features that would appear plausible in terms of gradual changes in the structure of the economy, for example associated with technological and financial innovation, or cultural and institutional changes. First, structural parameter changes are small over short horizons of a month or a year. Second, changes in structural parameters gradually build over time and can be significant over long horizons of many years or decades. Parameters therefore can change much more in the long run than the short run, generating substantial uncertainty about the level of parameters, even though monthly or annual changes are small.

\footnotetext{
${ }^{13}$ These risk-premia shocks are assumed to be uncorrelated with the observed fundamentals $\Delta f_{n t}$, which exogenously generates a disconnect between $\Delta s_{t}$ and the observed fundamentals. For a more endogenous explanation of the disconnect between exchange rates and observed fundamentals, related to private information, see Bacchetta and van Wincoop (2006).
} 
In order to get these features, we set $\theta_{1}=1$ and then choose the other parameters $\theta_{i}(i=2, . ., T)$ such that we maximize the ratio of the unconditional standard deviation of $\beta_{t}$ relative to the standard deviation of monthly changes in $\beta_{n t}$. In other words we maximize

$$
\frac{\sigma_{\beta_{n t}}}{\sigma_{\Delta \beta_{n t}}}
$$

The resulting process implies that an innovation impacts the parameter $\beta_{n t}$ slowly over time in the form of a hump shape. It builds up to a maximum impact after $T / 2$ periods and then gradually declines. We will examine other processes in section 3.3.

Table 1 reports the parameters adopted for the benchmark parameterization. The first four parameters relate to the processes for $\beta_{n t}$. We set $T=1000$. Since we assume that one period is one month, this implies that the current level of structural parameters is determined by parameters innovations over the last 1000 months or 83 years. We set $N=5$, so that the total number of structural parameters (and fundamentals) is 5. Therefore the total number of unknown structural parameter innovations that agents need to learn about is 5000 . We normalize by setting the mean value of the parameters at $\beta=1$. We set $\sigma_{\beta}=0.000165$. As reported in the last row of Table 2 , this implies a monthly standard deviation of the change in $\beta_{n t}$ of $0.3 \%$ of the mean value of parameters, which is small. But there is considerable uncertainty about the level of parameters as their unconditional standard deviation is 1.2 , or $120 \%$ of their steady state level. This is because parameter changes build gradually over time.

The next five parameters are associated with the process for $b_{t}$ and $v_{t}$. These are set to closely match four moments related to exchange rates and interest rates: the standard deviation of $\Delta s_{t}$, the standard deviation of $i_{t}-i_{t}^{*}$, the first-order autocorrelation of $\Delta s_{t}$ and the first-order autocorrelation of $i_{t}-i_{t}^{*}$. In doing so, we use monthly data from 1975(9) to 2008(9) for exchange rates and interest differentials of 5 countries relative to the United States. The countries are Canada, Germany, Japan, Switzerland, and United Kingdom. These moments are reported in the first column of Table 2 (first 4 rows). We match these moments in the model for the case of constant parameters $\left(\sigma_{\beta}=0\right)$. The moments for constant parameters are reported in the second column of Table 2 . But the moments are virtually identical under the benchmark assumption about time-varying parameters, as shown 
in column $3 .^{14}$

As a by-product the model also generates a significant negative correlation between the change in the exchange rate and lagged interest differential. The Fama regression coefficient, reported in the fifth row of Table 2, is even slightly more negative than in the data. We emphasize that this is not intended as an explanation for the forward discount puzzle as it is due to entirely exogenous risk-premium shocks (see Bacchetta and van Wincoop (2009) for a more plausible explanation for the forward discount puzzle). It does imply though that the model is well grounded in the data as it conforms to the basic statistical properties of exchange rates and interest rates.

The next two parameters relate to the process of the observed fundamentals. We set the number of fundamentals at $N=5$. We do not take a strong stand on exactly which observed fundamentals affect exchange rates. This is not necessary as the finding that observed fundamentals have limited explanatory power for exchange rates is well known and applies broadly across fundamentals. But for concreteness in terms of the calibration, we use some representative results from Bacchetta, van Wincoop and Beutler (2009). For the same 5 currencies and sample period used to calibrate exchange rate and interest rate moments, they regress $\Delta s_{t}$ on changes in 5 fundamentals ( $\Delta f_{n t}$ in our model): changes in money supply, industrial production, unemployment rate, and oil price and the level of lagged interest rates. They obtain an average $R^{2}$ of 0.023 .

We set the standard deviation $\sigma_{f}$ of fundamental innovations in the model equal to $0.125 \%$ in order to match the average $R^{2}$ in the data when computed over a sample of 397 months (33 years) that corresponds to the sample in the data. As shown in Table 2, we match this for both constant parameters and the benchmark assumption of time-varying parameters. We set the persistence $\rho_{f}$ of the process for fundamentals equal to 0 under the benchmark parameterization. This is also closely consistent with the specific fundamentals listed above. ${ }^{15} \mathrm{We}$

\footnotetext{
${ }^{14}$ Both under constant and time-varying parameters the moments are computed based on a simulation over 1300 months (108 years). So they can reasonably be considered population moments. Prior to the 1300 months over which we compute the moments we first simulate the model for $T=1000$ months (83 years) in order to obtain a history.

${ }^{15}$ The change in money supply, industrial production, unemployment rate, and the oil price all have low persistence, with first-order autocorrelations averaging to 0.02 . Only the lagged interest rate differential has a high persistence of 0.94 .
} 
will also consider positive persistence in sensitivity analysis.

Finally, we set $\alpha=100 / 3$, implying a discount rate $\lambda$ in the present value equation for the exchange rate of 0.97. This is consistent with evidence by Engel and West (2005) that the discount rate is close to 1.

\subsection{Results}

We simulate the model over 2300 months. All moments reported drop the first 1000 months in order to generate a prior history of shocks. Unless otherwise indicated, the results reported are based on the subsequent 1300 months.

\section{Derivative of Exchange Rate with Respect to Fundamentals}

Figures 1 and 2 show $\partial \Delta s_{t} / \partial \Delta f_{n t}$ for each of the five fundamentals. From now on we simply refer to this as the derivative of the exchange rate with respect to fundamentals. Figure 1 does so for a 10-year period (observations 1540-1659 in the simulation), while Figure 2 does so for a 100-year period (observations 1001-2200 in the simulation). ${ }^{16}$ Both Figures also show $\beta_{n t}$, which would be the derivative of the exchange rate with respect to fundamentals if parameters were known.

It is evident from Figure 1 that the derivative of the exchange rate with respect to fundamentals is far more volatile than the underlying structural parameters. As reported in Table 2, the average standard deviation of monthly changes in the derivative is $25.9 \%$ of the mean value of the derivative. By contrast, the standard deviation of monthly changes in the underlying structural parameters is only $0.3 \%$. We will call the ratio between these two standard deviations the "scapegoat ratio" as scapegoat effects are responsible for the increased instability in the relationship between the exchange rate and fundamentals. In the benchmark case, this ratio is equal to 85.1.

This result is consistent with the results from the order analysis in section 2.5, which shows that the actual structural parameter innovations have very limited effect on the derivative. Instead, we saw in section 2.5 that the derivative is very much driven by noise innovations $\varepsilon_{t}^{b}$, which affect the expectations of parameters in conjunction with changes in observed fundamentals through scapegoat effects.

While Figure 1 would suggest that the derivative of exchange rates with respect

\footnotetext{
${ }^{16}$ Figure 1 corresponds to the middle observations of Figure 2.
} 
to fundamentals is entirely disconnected from the true underlying structural parameters, Figure 2 shows that this is not the case when we take a longer 100-year view. There are large changes in parameters over long cycles of several decades, while the derivative of the exchange rate with respect to the fundamentals broadly catches up with these long term swings. This implies that when there are persistent changes in parameters, agents do eventually learn about them when they are consistently reflected in the data $F_{t}+b_{t}$ for several decades.

But, as illustrated in both Figures 1 and 2, short to medium-term fluctuations around such long-term cycles can be large and even dominate the trend itself. It is precisely the possibility that parameters can change a lot in the long run that creates significant uncertainty about their level and gives rise to scapegoat effects that lead to large changes in the derivatives over the short to medium run.

\section{Expectation of Parameters}

It is useful to recall equation (19) of the derivative of the exchange rate with respect to fundamentals, which is displayed here again for convenience:

$$
\frac{\partial \Delta s_{t}}{\partial \Delta f_{n t}}=(1-\lambda) \beta_{n t}+\lambda E_{t} \beta_{n t}+\lambda \sum_{i=0}^{T} \Delta \mathbf{f}_{t-i}^{\prime} \frac{\partial E_{t} \boldsymbol{\beta}_{t-i}}{\partial \Delta f_{n t}}
$$

Since $\lambda$ is close to 1 , the derivative of the exchange rate with respect to fundamentals is primarily driven by the last two terms. The second term is proportional to the expectation $E_{t} \beta_{n t}$ of structural parameter $n$. Focusing on variable 1 , Figure 3 compares the evolution of $\beta_{1 t}$ with $E_{t} \beta_{1 t}$ over the samples of 10 and 100 years used in Figures 1 and 2. The top panels illustrate that $E_{t} \beta_{1 t}$ is significantly more volatile than the underlying parameter $\beta_{1 t}$. But a comparison with Figures 1 and 2 also shows that the overall derivative $\partial \Delta s_{t} / \partial \Delta f_{n t}$ has even much larger fluctuations at high frequencies. This is illustrated in the bottom panels of Figure 3 , which show $\beta_{1 t}, E_{t} \beta_{1 t}$ as well as $\partial \Delta s_{t} / \partial \Delta f_{1 t}$.

It follows that the very high frequency volatility in $\partial \Delta s_{t} / \partial \Delta f_{n t}$ is caused by the last term in (32). This confirms the analysis in section 2.5 when breaking the derivative into components of different orders. As explained in section 2.5, the last term in (32) has a second-order component that is proportional to $\varepsilon_{t}^{b}$ (zero and first-order components are zero). Therefore, this term has no persistence and gives rise to very high frequency fluctuations. By contrast, $E_{t} \beta_{n t}$ generates more 
persistent fluctuations in the derivative that deviate from the actual structural parameter.

To summarize, very gradual changes in structural parameters can lead to a highly unstable relationship between exchange rates and observed fundamentals. Three factors play a role in this regard. First, scapegoat effects lead to high frequency fluctuations in the derivative of the exchange rate with respect to fundamentals due to transitory changes in parameter expectations resulting from changes in observed fundamentals. Second, scapegoat effects also lead to more persistent changes in the derivative due to persistent changes in the expected value of structural parameters that are unrelated to the actual changes in structural parameters. Finally, at a very low frequency the derivative also picks up changes in the structural parameters themselves.

\subsection{Sensitivity to Process of Structural Parameters}

Perhaps most relevant when conducting sensitivity analysis with respect to these findings is to consider how the results depend on the process of parameters. This is the only aspect of the model that we could not calibrate to the data. There are good reasons for this. It is impossible to know what exactly the process of structural parameters is. As emphasized in the Introduction, econometric analysis cannot distinguish between lots of different processes. Nonetheless it is important to consider alternative processes. We will do so in order to make a general point, which is key to our results. There is significant reduced-form parameter instability relative to structural parameter instability when structural parameters can potentially change much more in the long-run than the short-run. This implies significant uncertainty about the level of parameters relative to monthly changes

in parameters. Or in more technical terms, for any process where $\sigma_{\beta_{n t}} / \sigma_{\Delta \beta_{n t}}$ is high, there will be a high scapegoat ratio.

In order to illustrate this point, we consider four alternative processes. These are all special cases of the process

$$
\beta_{n, t+1}-\beta_{n t}=\delta_{1}\left(\beta_{n t}-\beta_{n, t-1}\right)-\delta_{2}\left(\beta_{n t}-\beta\right)+\epsilon_{n t}
$$

with different values for $\delta_{1}$ and $\delta_{2}$. In terms of an MA process, (33) can be written 
as

$$
\beta_{n t}=\beta_{n}+\sum_{i=1}^{\infty} \theta_{i} \varepsilon_{n, t-i+1} .
$$

with $\theta_{1}=1, \theta_{2}=1+\delta_{1}-\delta_{2}$ and

$$
\theta_{i+1}-\theta_{i}=\delta_{1}\left(\theta_{i}-\theta_{i-1}\right)-\delta_{2} \theta_{i}
$$

for $i \geq 2$.

The benchmark process is a special case of this process as well, with $\delta_{1}=1$ and $\delta_{2}=0.00000985$. The benchmark process truncates the $M A$ process to an $M A(T)$ by setting $\theta_{i}=0$ for $i>T$. The alternative Process 1 is different in that we do not truncate. As is the case for the benchmark process, the parameters $\theta_{i}$ are chosen to maximize the standard deviation of $\tilde{\beta}_{n t}$ relative to $\Delta \beta_{n t}$, where $\tilde{\beta}_{n t}=\sum_{i=1}^{T} \theta_{i} \epsilon_{n, t-i+1}$ captures the component of $\beta_{n t}$ that is unknown at time $t$ (most recent $T$ innovations). But the coefficients $\theta_{i}$ are not restricted to be zero for $i>T$. In the alternative Processes 2 through 4 we truncate $\theta_{i}=0$ for $i>T$ as for the benchmark process.

For all 4 alternative processes, Figure 4 shows the impulse response functions of the structural parameters after a one standard deviation parameter innovation. For comparison each chart also shows the impulse response function for the benchmark parameterization. The top of each chart shows the parameters $\delta_{1}$ and $\delta_{2}$ for each of the alternative processes. In each case the standard deviation of $\tilde{\beta}_{n t}$ is kept the same as under the benchmark parameterization.

In Process 1 the structural parameter rises gradually in response to an innovation, until it peaks at a new level where it will remain. From a theoretical standpoint this process has the unattractive feature that the structural parameters are non-stationary and therefore unbounded. But in practice we only simulate the model over a finite 2300 months (192 years) and the uncertainty of $\beta_{n t}$ due to parameter innovations over the past $T=1000$ months (83 years) is kept identical across all parameterizations. This process captures the idea that certain structural changes, such as technological and financial innovation, are indeed permanent. It also connects well to a lot of the econometrics literature that tests for structural breaks in parameters. This amounts to testing for permanent changes in parameters. In a way Process 1 captures even better than the benchmark parameterization what we have in mind with gradual and long-lasting changes in parameters. We 
only chose to truncate the benchmark process after $T$ innovations in order to assure stationarity for theoretical reasons. ${ }^{17}$

Process 2 is a truncated AR(1) process with AR coefficient of 0.99 . Process 3 is a truncated random walk process. In Process 4 the structural parameter gradually rises over time in response to an innovation and peaks a bit earlier than under the benchmark. In these three cases the response is truncated to zero after $T$ periods.

Table 3 shows the scapegoat ratio for each of the processes, as well as $\sigma_{\tilde{\beta}_{n t}} / \sigma_{\Delta \beta_{n t}}$. Since $\sigma_{\tilde{\beta}_{n t}}$ is kept the same across all processes, a higher ratio means a smaller standard deviation $\sigma_{\Delta \beta_{n t}}$ of monthly changes in structural parameters. Table 3 clearly shows that the higher the long-run uncertainty about the level of the structural parameters relative to monthly changes in structural parameters, the bigger the scapegoat ratio. For Process 1, where $\sigma_{\tilde{\beta}_{n t}} / \sigma_{\Delta \beta_{n t}}$ is about double that under the benchmark process, the scapegoat ratio is now an amazing 267.7. This is more than three times that under the benchmark. Figure 5 illustrates the scapegoat effect in this case. A major difference is that structural parameters are more stable, even at very low frequency. Both the expectation of $\beta_{n t}$ and the reduced form parameters $\partial \Delta s_{t} / \partial \Delta f_{n t}$ remain highly unstable and are now even more disconnected from the smooth structural parameters.

By construction $\sigma_{\tilde{\beta}_{n t}} / \sigma_{\Delta \beta_{n t}}$ is less for the other processes than under the benchmark parameterization and therefore the scapegoat ratio is lower as well. ${ }^{18}$ Beyond that, two points are worth making. First, even when $\sigma_{\tilde{\beta}_{n t}} / \sigma_{\Delta \beta_{n t}}$ is much lower than under the benchmark parameterization, there can still be a substantial scapegoat ratio. For example, for Process 4 , where $\sigma_{\tilde{\beta}_{n t}} / \sigma_{\Delta \beta_{n t}}$ is less than one sixth that under the benchmark, the reduced form monthly parameter instability is still more than 10 times the structural parameter instability. Second, even when the scapegoat ratio is close to 1 , as it is for Process 2, this does not mean that that reduced form parameters are similar to structural parameters. Indeed, even for Process 2 , the correlation between monthly changes in structural parameters and reduced form parameters is only 0.33 (it is 0.02 in the benchmark case).

\footnotetext{
${ }^{17}$ Of course one could truncate process 1 after $\bar{T}$ innovations, with $\bar{T}$ much larger than $T$. The results will then be very similar to what we report for Process 1 even though technically the process will then be stationary.

${ }^{18}$ Recall that the benchmark process is chosen to maximize $\sigma_{\tilde{\beta}_{n t}} / \sigma_{\Delta \beta_{n t}}$ for processes that are truncated after $T$ periods.
} 
We should finally point out that we have restricted ourselves to processes with normally distributed innovations. It is possible that some parameter changes are big and infrequent. One can imagine a process where there is a big change in parameters with some very small probability $p>0$. In that case parameters are perfectly constant almost all of the time. But even when structural parameters do not change at all, reduced-form parameters will be very volatile as the infrequent large parameter changes contribute to significant uncertainty about the level of parameters. ${ }^{19}$

\subsection{Other Sensitivity Analysis}

We now return to the benchmark process and examine the extent to which the results are sensitive to changes in various parameters. We consider four types of parameters: the standard deviation of structural parameter innovations; the variability and persistence of fundamentals; the horizon $T$ after which parameters are known; and the volatility of the unobserved fundamentals.

\subsubsection{Sensitivity Moments to Parameter Instability}

When considering alternative processes for the parameters in the previous subsection, we held constant the overall parameter instability as measured by the standard deviation of $\tilde{\beta}_{t}$. We now consider the impact of a change in the standard deviation $\sigma_{\beta}$ of parameter innovations for the benchmark process.

Table 2 reports moments for three values of $\sigma_{\beta}$. In addition to the constant parameter and the benchmark time-varying parameter cases, the fourth column shows the case where the standard deviation of parameter innovations is twice that under the benchmark $\left(\sigma_{\beta}=0.00033\right)$. In the latter case the standard deviation of monthly changes in the derivative of the exchange rate with respect to fundamentals is $45 \%$, while the same moment is only $0.6 \%$ for the structural parameters. This implies a scapegoat ratio of 73.8 . While this remains very high, it is slightly lower than under the benchmark parameterization. The reason for this

\footnotetext{
${ }^{19}$ For example, when the structural parameter follows a Markov process with two states $1+a$ and $1-a$ and the probability of changing from one state to another is a small $p$, then $\sigma_{\tilde{\beta}_{n t}} / \sigma_{\Delta \beta_{n t}}$ is equal to $1 /(4 p)$. This can get very large for small $p$. Our results from Table 3 suggest that this will again generate a very large scapegoat ratio.
} 
is that when structural parameters become sufficiently volatile, it becomes easier to learn about them through data on $F_{t}+b_{t}$. This reduces the rational confusion and associated scapegoat effects, although numerically the difference is small.

Even though we have seen that gradual changes in structural parameters lead to a highly unstable relationship between exchange rates and fundamentals, some basic moments involving exchange rates and interest rates are remarkably insensitive to the degree of parameter instability. Exchange rate volatility rises only slightly. The standard deviation of exchange rate changes rises from $2.90 \%$ to $3.04 \%$, from the case of constant parameters to the extreme case where parameter volatility is twice that under the benchmark. The standard deviation of the interest rate differential, as well as the autocorrelation of monthly exchange rate change and the interest differential, are all virtually unaffected by parameter volatility. The same is the case for the monthly Fama regression coefficient of $\Delta s_{t+1}$ on $i_{t}-i_{t}^{*}$. The reason for these results is that most exchange rate volatility is unrelated to changes in fundamentals. For the benchmark parameterization the $R^{2}$ is 0.023 , as in the data.

\subsubsection{Sensitivity to Process Fundamentals}

We first examine the impact of the fundamentals process on the link between exchange rates and these fundamentals. We consider a higher standard deviation of the innovations of the fundamentals and positive persistence of changes in the fundamentals. We find that the volatility of $\frac{\partial \Delta s_{t}}{\partial \Delta f_{n t}}$ decreases with $\sigma_{f}$. When we set the standard deviation of innovations four times as large as under the benchmark $\left(\sigma_{f}=0.005\right)$, the scapegoat ratio declines from 85.1 to 53.7 .

The explanation for these results is that when $\sigma_{f}$ is larger, the signal $F_{t}+b_{t}$ becomes more informative about structural parameters as they are multiplied by fundamentals that fluctuate more. Consequently, there is less confusion. Scapegoat effects are smaller and therefore the derivative $\frac{\partial \Delta s_{t}}{\partial \Delta f_{n t}}$ is somewhat less volatile. We should not overstate this though as monthly changes in this derivative remain 54 times more volatile than monthly change in the structural parameter $\beta_{n t}$. Moreover, a standard deviation of $\sigma_{f}=0.005$ is implausibly high as it leads to an $R^{2}$ of 0.15. This is well above representative results for a sample of at least 3 decades.

We also consider raising the persistence $\rho_{f}$ of $\Delta f_{n t}$ from 0 to 0.2 . As shown in 
Appendix A, the derivative of exchange rates with respect to fundamentals is then also affected by expectations of future levels of the structural parameters. But the overall impact on the unstable relationship between exchange rates and observed fundamentals is small. The scapegoat ratio increases slightly from 85.1 to 96.7.

\subsubsection{Sensitivity to the horizon $\mathrm{T}$}

A smaller $T$ implies that there are fewer parameter innovations to learn about. This reduces rational confusion and scapegoat effects. This is illustrated by comparing the case of $T=1000$ to the case of $T=300$. For $T=300$ we find a scapegoat ratio of 9.3. While this still reflects significant scapegoat effects, it is much smaller than scapegoat ratio of 85.1 found in the benchmark of $T=1000$. Conversely, the scapegoat ratio would rise as we make $T$ even bigger than 1000 . However, this would take an excessive amount of computer time. With $T=1000,5$ fundamentals and a simulation over 2300 months we already need to solve 2300 signal extraction problems that each involve 5000 unknown parameter innovations. ${ }^{20}$

\subsubsection{Sensitivity to $\sigma_{b}$ (volatility of unobserved fundamentals)}

Shocks to unobserved fundamentals play a crucial role in generating scapegoat effects. However, there is a non-linear relationship between the volatility of unobserved fundamentals and the magnitude of the scapegoat effect as measured by the scapegoat ratio. This is illustrated in Figure 6, which plots the scapegoat ratio as a function of $\sigma_{b}$. As the standard deviation $\sigma_{b}$ of the unobserved fundamentals rises, the scapegoat ratio first increases and then eventually starts to fall. This non-linear relationship can be explained by the inference process. At low values, an increase in $\sigma_{b}$ generates more rational confusion as $F+b$ becomes more volatile. But when $b$ becomes too volatile, $F+b$ is a less valuable source of information for investors. They will then attach less weight to it when forming expectations about parameters, which reduces scapegoat effects.

\footnotetext{
${ }^{20}$ With our current technology, this takes about 40 hours of computer time.
} 


\section{Time-Varying Coefficients and Forecasting Per- formance}

The previous section has shown that a significantly unstable relationship between exchange rates and fundamentals results from gradual changes in structural parameters coupled with the unobservability of the structural parameters. In this section we investigate the implications of this unstable relationship for out-of-sample forecasting and the corresponding Meese-Rogoff puzzle. If parameters were known, whether constant or time varying, by construction the model would outperform a random walk in predicting exchange rates. Since the empirical evidence shows that this is not the case, one can only conclude that this is due to the estimation error of the parameters levels. One might expect this problem to become more severe when parameters are time varying. This has led Meese and Rogoff (1983a,b) and many others to conjecture that time-varying parameters may be responsible for the poor out-of-sample forecasting performance of the model relative to the random walk. In this section we evaluate the validity of this conjecture within the context of our model. A companion paper, Bacchetta, van Wincoop and Beutler (2009) provides further insight by considering the implications of exogenous reduced form parameter instability in the exchange rate equation.

\subsection{Out-of-Sample Forecasting in the Data}

\subsubsection{The Meese-Rogoff experiment}

In their seminal paper, Meese and Rogoff (1983a) conduct an out-of-sample forecasting exercise. It is not true forecasting as they forecast the future exchange rate using information about future macro fundamentals. The statistic they construct may be better called a measure of out-of-sample fit of the model. They first regress the exchange rate on a set of fundamental variables over a sample of $L$ months, using the first $L$ observations of their data. They use the estimate from this regression to compute a forecast at $L+1$, using the observed fundamentals at $L+1 .^{21}$ Using rolling regressions, they repeat this $P$ times, each time starting

\footnotetext{
${ }^{21}$ Meese and Rogoff (1983a) estimated the exchange rate equation in levels, using several lags of the exchange rate, but the subsequent literature has regressed the change in the exchange rate on fundamentals, sometimes including a cointegration term.
} 
the sample one month later. They then compute the ratio of the resulting Mean Square Error (MSE) with the one obtained assuming that the exchange rate follows a random walk. ${ }^{22}$ They assume $L=45$ and $P=55$, but subsequent studies have considered larger numbers for $L$ and $P$ as data samples became longer. For example, in Molodstova and Papell (2008) $L=108$ and $P=292$.

The key result of Meese and Rogoff (1983a) is that the MSE ratio is generally above 1, so that the average forecast error is larger when using the fundamentals than adopting a random walk assumption. This result has largely held up to extensive scrutiny in the more than two decades of research that followed. For example, Cheung, Chinn and Pascual (2005) consider a longer sample of data, more currencies, and more fundamental variables. In only 2 out of 216 combinations that they consider does the model significantly outperform the random walk at a $10 \%$ significance level. Rogoff and Stavrakeva (2008) discuss recent models that have been somewhat more successful but continue to find that the MSE ratio is generally above 1 or just slightly below 1 .

Figure 7 confirms this evidence. It is based on the same currencies, sample period and fundamentals used for the calibration in section 3.1. The currencies are the Canadian dollar, Japanese Yen, Swiss franc, British pound, and DM/euro. The fundamentals $\Delta f_{n t}$ are changes in money supply, industrial production, unemployment rate, and oil price and the level of lagged interest rates. Figure 7 shows the MSE ratio as we increase $L$ from 40 to 196 while keeping $P$ fixed at 200 . The results are reported for the average over the 5 exchange rates relative to the dollar. We see that the ratio is much higher than 1 when $L$ is small, and that it decreases towards 1 . However, while it gets close to 1 , it never goes below 1 . This confirms once again that the model does not beat the random walk.

\subsubsection{Small Sample Bias}

As discussed at the start of this section, the finding that the model underperforms the random walk must ultimately be due to parameter estimation error. If parameters were known, the model would by construction outperform the random

\footnotetext{
${ }^{22}$ More precisely, Meese and Rogoff (1983a) look at the RMSE which is the square root of MSE. They also look at the mean error and at the mean absolute error. They also consider the RMSE for forecasts further than 1 months ahead, in particular 6 and 12-month ahead forecasts.
} 
walk. One source of parameter estimation error is associated with small samples. This has been the focus of much recent literature. Estimating an exchange rate equation over a short data sample can lead to spurious noise in the estimation of $\beta$ even if it is constant. This can lead to a noisy forecast, raising the mean squared forecast error of the model compared to the random walk, which does not suffer from any estimation bias. This bias is also illustrated in Figure 7. The MSE ratio rises as $L$ falls and therefore the sample length for parameter estimation becomes smaller. This can indeed be a serious problem and statistics have been developed to correct for such small sample bias (e.g., Clark and West, 2006). However, even for relatively large values of $L$, involving more than two decades of data, it has been hard to outperform the random walk (e.g., Rogoff and Stavrakeva, 2008).

\subsection{The Effect of Time-Varying Parameters}

In order to investigate the relationship between time-varying parameters and the Meese-Rogoff results we compute the MSE ratio in the model for both constant and time-varying parameters. We do so for different values of $L$ and a large value of $P$ equal to 1000 . We first regress $\Delta s_{t}$ on the fundamentals $\Delta f_{n t}$. The first estimation sample uses observations $T+1$ to $T+L$ from the simulation. We use the estimated coefficients to predict $\Delta s_{T+L+1}$, using the actual future fundamentals $\Delta f_{n, T+L+1}$. We then use rolling regressions, as in Meese and Rogoff (1983a), with the last estimation sample using observations $T+P$ to $T+P+L-1$ from the simulation to predict $\Delta s_{T+P+L}$.

Figure 8 reports the results for $L$ ranging from 40 months to 200 months. Results are reported both for the benchmark parameterization with time-varying parameters and the case of constant parameters. It can be seen that the MSE ratio declines as the sample length $L$ increases, as in the data in Figure 7 . This illustrates the small sample bias that is largest for small sample lengths $L$.

A striking result emerges when we compare the MSE ratio of the time-varying coefficient model with the one of the constant coefficient model. We see that the forecasting performance is almost identical in the two cases. This result implies that time variation in the parameters is not a good explanation for the poor outof-sample forecasting performance of exchange rate models.

In Bacchetta, van Wincoop and Beutler (2009) we further explore what gives 
rise to this surprising result. We assume exogenous time-varying parameters in a reduced-form relationship between exchange rates and fundamentals. To be precise, we assume that $\Delta s_{t}=\sum_{n=1}^{N} \beta_{n t} \Delta f_{n t}+u_{t}$, with both the fundamentals $\Delta f_{n t}$ and reduced form parameters $\beta_{n t}$ following $\mathrm{AR}(1)$ processes. We find that three factors affect the MSE ratio of the time-varying parameter case relative to the constant parameter case.

The first two effects are substantial, but almost exactly offset each other unless the persistence of the reduced form parameters is close to 1. First, time-varying parameters raise the estimation error of the parameters. This comes on top of the small sample estimation error that also applies under constant parameters. Abstracting from small sample estimation error, the estimated parameters are a weighted average of the parameters during the estimation sample. This weighted average of parameters is generally different from the future parameters during the forecast horizon, causing additional parameter estimation error. This by itself raises the MSE ratio under time-varying parameters. It is also the reason why time-varying parameters have been suggested as a possible explanation for the Meese-Rogoff results.

But this is almost exactly offset by a second effect. Abstracting now from parameter estimation error, assume that the time-varying parameters are known. Time-varying parameters then increase the explanatory power of the fundamentals. Bacchetta et al. (2009) show that the explanatory power of the fundamentals is determined not by the mean value $\beta$ of the parameters, but by their expected squared value: $E_{t} \beta_{n t}^{2}$. This rises as a result of time-varying parameters. Intuitively, coefficients can occasionally take large values when they vary, thereby explaining more of the exchange rate fluctuations. This effect by itself lowers the MSE ratio under time-varying parameters.

Finally, there is a third effect that is quite small. The MSE for the random walk forecast is larger under time-varying parameters. This by itself lowers the MSE ratio under time-varying parameters. This can be seen in Table 2. The MSE for the random walk is equal to the variance of $\Delta s_{t}$. Table 2 shows that the volatility of $\Delta s_{t}$ rises with time-varying parameters. But it also shows that this effect is small.

Bacchetta et al. (2009) show that time-varying parameters only have a substantial effect on the MSE ratio in the extreme case where the persistence of the 
parameters in the reduced form relationship between exchange rates and fundamentals is close to 1 , or more precisely in the range of 0.95 to 1 . This is not the case under our benchmark parameterization, where the persistence is 0.86 . While the persistence of the structural parameters is close to 1 , the persistence of the reduced form parameters (derivative of $\Delta s_{t}$ with respect to $\Delta f_{n t}$ ) is reduced by fluctuations of high and intermediate frequencies associated with scapegoat effects.

If the persistence of the reduced form parameters is close to 1 , Bacchetta et al. (2009) show that the additional parameter estimation error due to time-varying parameters becomes small. With high persistence the weighted average of parameters during the estimation sample will be close to the parameters during the forecast horizon. Therefore the second factor described above dominates and timevarying parameters lower the MSE ratio. Even when this is the case, it goes in exactly the wrong direction in terms of explaining the Meese-Rogoff puzzle, which is about the high level of the MSE ratio in the data. In our view the puzzle is simply explained by the limited explanatory power of the observed fundamentals, together with small sample estimation error.

\section{Conclusion}

Anecdotal, survey and econometric evidence all suggest that the relationship between the exchange rate and macro fundamentals is highly unstable. One possible way to explain this is by assuming large and frequent known changes in the structural parameters. But this does not seem very plausible as structural parameters are hard to observe and estimate and many changes in the structure of the economy are gradual as a result of technological and financial innovation and institutional changes. We have therefore developed a model where structural parameters are not observed and changes in these structural parameters are very gradual. We have shown that the relationship between a forward looking variable like the exchange rate and macro fundamentals is determined not by the structural parameters themselves, but rather by the expectations of these structural parameters.

We have also shown that expectations of these parameters can change significantly and frequently, even when changes in structural parameters are small and gradual. This is a result of scapegoat effects, where changes in the exchange rate, or other macro data, are attributed to certain observed fundamentals even when 
they are driven by unobserved fundamental shocks. Such scapegoat effects occur in an environment where agents are rational Bayesian learners that incorporate all available information to revise their view on the parameters. When structural parameters can potentially change significantly over long horizons of several decades, there is substantial room for scapegoat effects as agents are trying to learn about the level of the parameters.

While our focus has been on the exchange rate, an analogous explanation could also account for the extensive evidence of parameter instability seen in other forward looking macroeconomic and financial data. Two key ingredients, which are not limited in any way to exchange rate models, drive our unstable reduced form results. First, there must be unobserved fundamental shocks. This applies surely to other asset prices as well and more generally to other macroeconomic data as factors driving business cycles and long term growth rates are not perfectly understood. Second, structural parameters must have the potential to change significantly over long horizons. This would be hard to dispute as well, especially in the context of major technological, financial and institutional changes over the past two centuries.

Separate from the question of what accounts for the time-varying relationship between exchange rates and observed fundamentals, there is also the question of what its implications are. The answer based on our findings is "very small". We have shown that even very large time variation in the relationship between exchange rates and fundamentals has little impact on the statistical properties of exchange rates, interest rates, the in-sample explanatory power of macro fundamentals and the ability to forecast out of sample. 


\section{Appendix}

\section{A Solving the General Model}

In this Appendix we describe the model's solution in the more general case, where the processes for $\Delta f_{n t}, b_{t}$, and $v_{t}$ are as specified in Section 3. A Technical Appendix provides further details towards the implementation of the simulations with Gauss. We start from the present value equation (1) of the exchange rate. We need to express it in way we can easily substitute the expectation terms. This equation can be rewritten as:

$$
s_{t}=(1-\lambda) F_{t}+(1-\lambda) b_{t}-\lambda v_{t}+(1-\lambda) \sum_{k=1}^{\infty} \lambda^{k} E_{t}\left(F_{t+k}+b_{t+k}\right)
$$

First, consider the term involving the present discounted value of $F$. Use that

$$
F_{t+k}=F_{t}+\sum_{n=1}^{N} \sum_{i=1}^{k} \beta_{n, t+i}\left(f_{n, t+i}-f_{n, t+i-1}\right)
$$

Therefore

$$
\sum_{k=1}^{\infty} \lambda^{k} F_{t+k}=\frac{\lambda}{1-\lambda} F_{t}+\frac{1}{1-\lambda} \sum_{n=1}^{N} \sum_{i=1}^{\infty} \lambda^{i} \beta_{n, t+i}\left(f_{n, t+i}-f_{n, t+i-1}\right)
$$

The present value of $b$ can be written as $\tilde{b} E_{t} b_{t}$, where

$$
\tilde{b}=(1-\lambda) \frac{\rho_{b} \lambda}{1-\rho_{b} \lambda}
$$

Using this, (36) becomes

$$
\begin{gathered}
s_{t}=(1-\lambda) F_{t}+\lambda E_{t} F_{t}+(1-\lambda) b_{t}-\lambda \nu_{t} \\
\sum_{n=1}^{N} \sum_{i=1}^{\infty}(\rho \lambda)^{i} E_{t} \beta_{n, t+i}\left(f_{n, t}-f_{n, t-1}\right)+\tilde{b} E_{t} b_{t}
\end{gathered}
$$

Therefore

$$
\begin{aligned}
& s_{t}-s_{t-1}=(1-\lambda) \sum_{n=1}^{N} \beta_{n t}\left(f_{n t}-f_{n, t-1}\right)+\lambda\left[E_{t} F_{t}-E_{t-1} F_{t-1}\right]+ \\
& \sum_{n=1}^{N} E_{t} \tilde{\beta}_{n t}\left(f_{n, t}-f_{n, t-1}\right)-\sum_{n=1}^{N} E_{t-1} \tilde{\beta}_{n, t-1}\left(f_{n, t-1}-f_{n, t-2}\right)+ \\
& (1-\lambda)\left(b_{t}-b_{t-1}\right)+\tilde{b}\left(E_{t} b_{t}-E_{t-1} b_{t-1}\right)-\lambda\left(v_{t}-v_{t-1}\right)
\end{aligned}
$$


where

$$
\tilde{\beta}_{n t}=\sum_{i=1}^{\infty}(\rho \lambda)^{i} \beta_{n, t+i}
$$

Finally, we can write

$$
\begin{aligned}
& E_{t} F_{t}-E_{t-1} F_{t-1}=E_{t}\left(F_{t}-F_{t-1}\right)+\left[E_{t} F_{t-1}-E_{t-1} F_{t-1}\right]= \\
& \sum_{n=1}^{N} E_{t} \beta_{n t}\left(f_{n t}-f_{n, t-1}\right)+\sum_{n=1}^{N} \sum_{i=1}^{T}\left(f_{n, t-i}-f_{n, t-i-1}\right)\left[E_{t} \beta_{n, t-i}-E_{t-1} \beta_{n, t-i}\right]
\end{aligned}
$$

Using (43) and collecting terms multiplying $f_{n t}-f_{n, t-1}$, (41) becomes

$$
\begin{aligned}
& s_{t}-s_{t-1}=\sum_{n=1}^{N}\left((1-\lambda) \beta_{n t}+\lambda E_{t} \beta_{n t}+E_{t} \tilde{\beta}_{n t}\right)\left(f_{n t}-f_{n, t-1}\right)+ \\
& -\sum_{n=1}^{N} E_{t-1} \tilde{\beta}_{n, t-1}\left(f_{n, t-1}-f_{n, t-2}\right)+ \\
& \lambda \sum_{n=1}^{N} \sum_{i=1}^{T}\left(f_{n, t-i}-f_{n, t-i-1}\right)\left[E_{t} \beta_{n, t-i}-E_{t-1} \beta_{n, t-i}\right]+ \\
& (1-\lambda)\left(b_{t}-b_{t-1}\right)+\tilde{b}\left(E_{t} b_{t}-E_{t-1} b_{t-1}\right)-\lambda\left(v_{t}-v_{t-1}\right)
\end{aligned}
$$

Given the processes of $\boldsymbol{\beta}_{t}$ and $b_{t}$, the terms including expectations can be written as:

$$
\begin{aligned}
& E_{t} \beta_{n t}-\beta=\hat{\omega} E_{t} \boldsymbol{\xi}_{n t} \\
& E_{t} \tilde{\beta}_{n t}-\frac{\rho \lambda}{1-\rho \lambda} \beta=\hat{\theta} E_{t} \boldsymbol{\xi}_{n t} \\
& E_{t} b_{t}=\hat{b} E_{t} \mathbf{b}_{t}+\rho_{b}^{T} b_{t-T} \\
& \sum_{i=1}^{T}\left(f_{n, t-i}-f_{n, t-i-1}\right)\left[E_{t} \beta_{n, t-i}-E_{t-1} \beta_{n, t-i}\right]= \\
& \quad \sum_{i=1}^{T}\left(f_{n, t-i}-f_{n, t-i-1}\right) \theta_{T-i+1} \epsilon_{n, t-T}+\hat{h}_{n t} E_{t} \boldsymbol{\xi}_{n t}-\hat{f}_{n, t-1} E_{t-1} \boldsymbol{\xi}_{n, t-1}
\end{aligned}
$$

where $\bar{\theta}, \hat{\theta}, \hat{b}, \hat{h}$ and $\bar{h}$ are 1 by $T$ vectors with

$$
\begin{aligned}
\hat{\omega}(j) & =\theta_{j} \\
\hat{\theta}(j) & =\sum_{i=1}^{T-j} \theta_{j+i}(\rho \lambda)^{i} \\
\hat{b}(j) & =\rho_{b}^{j-1}
\end{aligned}
$$




$$
\begin{aligned}
& \hat{h}_{n t}(j)=\sum_{i=1}^{j-1}\left(f_{n, t-i}-f_{n, t-i-1}\right) \theta_{j-i} \\
& \hat{f}_{n, t-1}(j)=\sum_{i=1}^{j}\left(f_{n, t-i}-f_{n, t-i-1}\right) \theta_{j-i+1}
\end{aligned}
$$

and $\hat{h}_{n t}(1)=0$.

Substituting these results into (44) gives

$$
\begin{aligned}
& s_{t}-s_{t-1}=\sum_{n=1}^{N}\left(\frac{\beta}{1-\rho \lambda}+(1-\lambda)\left(\beta_{n t}-\beta\right)+[\lambda \hat{\omega}+\hat{\theta}] E_{t} \boldsymbol{\xi}_{n t}\right)\left(f_{n t}-f_{n, t-1}\right)+ \\
& -\sum_{n=1}^{N}\left(\frac{\rho \lambda \beta}{1-\rho \lambda}+\hat{\theta} E_{t-1} \boldsymbol{\xi}_{n, t-1}\right)\left(f_{n, t-1}-f_{n, t-2}\right)+ \\
& \lambda \sum_{n=1}^{N} \sum_{i=1}^{T}\left(f_{n, t-i}-f_{n, t-i-1}\right) \theta_{T-i+1} \epsilon_{n, t-T}+\lambda \sum_{n=1}^{N}\left(\hat{h}_{n t} E_{t} \boldsymbol{\xi}_{n t}-\hat{f}_{n, t-1} E_{t-1} \boldsymbol{\xi}_{n, t-1}\right)+ \\
& (1-\lambda)\left(b_{t}-b_{t-1}\right)-\lambda\left(v_{t}-v_{t-1}\right)+\tilde{b}\left(\hat{b}\left(E_{t} \mathbf{b}_{t}-E_{t-1} \mathbf{b}_{t-1}\right)+\rho_{b}^{T}\left(b_{t-T}-b_{t-T-1}\right)\right)
\end{aligned}
$$

The expectation terms can be derived from the signal extraction problem, where $E_{t} \boldsymbol{\omega}_{t}=\mathbf{C}_{t} \boldsymbol{\omega}_{t}$. This gives:

$$
\begin{aligned}
& s_{t}-s_{t-1}=\sum_{n=1}^{N}\left(\frac{\beta}{1-\rho \lambda}+(1-\lambda)\left(\beta_{n t}-\beta\right)+\left[\lambda \bar{\omega}^{n}+\bar{\theta}^{n}\right] \mathbf{C}_{t} \boldsymbol{\omega}_{t}\right)\left(f_{n t}-f_{n, t-1}\right)+ \\
& -\sum_{n=1}^{N}\left(\frac{\rho \lambda \beta}{1-\rho \lambda}+\bar{\theta}^{n} \mathbf{C}_{t-1} \boldsymbol{\omega}_{t-1}\right)\left(f_{n, t-1}-f_{n, t-2}\right)+ \\
& \lambda \sum_{n=1}^{N} \sum_{i=1}^{T}\left(f_{n, t-i}-f_{n, t-i-1}\right) \theta_{T-i+1} \epsilon_{n, t-T}+\lambda \sum_{n=1}^{N}\left(\bar{h}_{t}^{n} \mathbf{C}_{t} \boldsymbol{\omega}_{t}-\bar{f}_{t-1}^{n} \mathbf{C}_{t-1} \boldsymbol{\omega}_{t-1}\right)+ \\
& (1-\lambda)\left(b_{t}-b_{t-1}\right)-\lambda\left(v_{t}-v_{t-1}\right)+\tilde{b}\left(\bar{b}\left(\mathbf{C}_{t} \boldsymbol{\omega}_{t}-\mathbf{C}_{t-1} \boldsymbol{\omega}_{t-1}\right)+\rho_{b}^{T}\left(b_{t-T}-b_{t-T-1}\right)\right)
\end{aligned}
$$

Here $\bar{\theta}^{n}$ is a 1 by $(N+1) T$ vector with $\hat{\theta}$ in elements $T(n-1)+1$ through $T n$ and zeros otherwise. The vectors $\bar{\omega}^{n}, \bar{h}_{t}^{n}$ and $\bar{f}_{t-1}^{n}$ are defined analogously. $\bar{b}$ is a 1 by $(N+1) T$ vector with $\hat{b}$ in elements $N T+1$ through $N T+T$ and zeros otherwise.

Collecting terms in $\mathbf{C}_{t} \boldsymbol{\omega}_{t}$ and $\mathbf{C}_{t-1} \boldsymbol{\omega}_{t-1}$, we can rewrite this as

$$
\begin{aligned}
& s_{t}-s_{t-1}=\sum_{n=1}^{N}\left(\frac{\beta}{1-\rho \lambda}+(1-\lambda)\left(\beta_{n t}-\beta\right)\right)\left(f_{n t}-f_{n, t-1}\right)+ \\
& \left(\sum_{n=1}^{N}\left[\lambda \bar{\omega}^{n}+\bar{\theta}^{n}\right]\left(f_{n t}-f_{n, t-1}\right)+\lambda \sum_{n=1}^{N} \bar{h}_{t}^{n}+\tilde{b} \bar{b}\right) \mathbf{C}_{t} \boldsymbol{\omega}_{t}-
\end{aligned}
$$




$$
\begin{aligned}
& \left(\sum_{n=1}^{N} \bar{\theta}^{n}\left(f_{n, t-1}-f_{n, t-2}\right)+\lambda \sum_{n=1}^{N} \bar{f}_{t-1}^{n}+\tilde{b} \bar{b}\right) \mathbf{C}_{t-1} \boldsymbol{\omega}_{t-1}+ \\
& -\sum_{n=1}^{N} \frac{\rho \lambda \beta}{1-\rho \lambda}\left(f_{n, t-1}-f_{n, t-2}\right)+\lambda \sum_{n=1}^{N} \sum_{i=1}^{T}\left(f_{n, t-i}-f_{n, t-i-1}\right) \theta_{T-i+1} \epsilon_{n, t-T}+ \\
& (1-\lambda)\left(b_{t}-b_{t-1}\right)+\tilde{b} \rho_{b}^{T}\left(b_{t-T}-b_{t-T-1}\right)-\lambda\left(v_{t}-v_{t-1}\right)
\end{aligned}
$$

The derivative with respect to the current fundamental is:

$$
\begin{aligned}
& \partial \Delta s_{t} / \partial \Delta f_{n t}=\left(\frac{\beta}{1-\rho \lambda}+(1-\lambda)\left(\beta_{n t}-\beta\right)\right)+ \\
& \frac{\partial\left(\sum_{n=1}^{N}\left[\lambda \bar{\omega}^{n}+\bar{\theta}^{n}\right]\left(f_{n t}-f_{n, t-1}\right)+\lambda \sum_{n=1}^{N} \bar{h}_{t}^{n}+\tilde{b} \bar{b}\right) \mathbf{C}_{t} \boldsymbol{\omega}_{t}}{\partial \Delta f_{n t}}
\end{aligned}
$$

\section{B Signal Extraction}

The signal extraction problem is described in Section 2.3. The matrix $\mathbf{H}_{t}$ is defined as:

$$
\mathbf{H}_{t}^{\prime}=\left[\mathbf{A}_{1 t}, \ldots, \mathbf{A}_{N t}, \mathbf{B}\right]
$$

with

$$
\mathbf{A}_{n t}=\left[\begin{array}{cccc}
\hat{f}_{n t}(1) & \hat{f}_{n t}(2) & \ldots & \hat{f}_{n t}(T) \\
0 & \hat{f}_{n, t-1}(1) & \ldots & \hat{f}_{n, t-1}(T-1) \\
& \ldots & \ldots & \ldots \\
0 & 0 & \ldots & \hat{f}_{n, t-T+1}(1)
\end{array}\right]
$$

and

$$
\mathbf{B}=\left[\begin{array}{cccc}
1 & \rho_{b} & \ldots & \rho_{b}^{T-1} \\
0 & 1 & \ldots & \rho_{b}^{T-2} \\
& \ldots & \ldots & \ldots \\
0 & 0 & \ldots & 1
\end{array}\right]
$$

\section{Empirical Evidence on Meese-Rogoff}

As in Meese-Rogoff (1983a), we implement rolling regressions to produce the outof-sample forecasts underlying the MSE ratios in Figure 7. The forecasts are based on the following regression:

$$
\Delta s_{t}=\Delta \mathbf{f}_{t}^{\prime} \mathbf{a}+u_{t}
$$


where $\mathbf{a}$ is the vector of constant parameters to be estimated and $\Delta=(1-L)$, where $L$ is the lag operator. The size of the estimation sample is $L$ and the forecasting sample size is $P$, hence the regression is rolled forward $P$ times. The $M S E^{m}$ is given by

$$
M S E^{m}=\frac{1}{P} \sum_{t=L}^{L+P-1}\left(\Delta s_{t+1}-\Delta \mathbf{f}_{t+1}^{\prime} \widehat{\mathbf{a}}\right)^{2}
$$

where $\widehat{\mathbf{a}}$ is estimated by OLS over the interval $t+1-L$ to $t$. Realized values of $\mathbf{f}_{t+1}$ are used to compute the forecast of $\Delta s_{t+1}$.

We compute a similar measure $M S E^{r w}$ for the same forecasting sample, assuming the exchange rate follows a random walk, i.e. setting $\widehat{\mathbf{a}}=0$ in the above equation. $M S E^{m}$ is divided by $M S E^{r w}$ to obtain the ratio of MSE displayed in Figure 7 . We set $P=200$ and vary $L$ from 40 to 196 . We compute the MSE ratios and take the simple average over the five countries mentioned in Figure 7.

The exchange rate, $s_{t}$, is the bilateral US Dollar end-of-period rate from IFS. The vector $\Delta \mathbf{f}_{t}^{\prime}$ is made of a constant and the following 5 variables:

- Money supply: $\Delta\left(m_{t}-m_{t}^{U S}\right)$, where $m_{t}=\ln M_{t}$ and $M_{t}$ is M1, OECD Main Economic Indicators (MEI), for Canada and M1, IFS line 59MA, for Japan. In the case of Germany/Euro area, we consider M1 seasonally adjusted, IFS line 59MACZF until December 1998 and M1, OECD MEI, for the Euro Area from January 1999. For the United Kingdom, we take M0, IFS line 19MC.ZF, until April 2006 (last observation of the IFS series) and M1, OECD MEI, from May 2006. For Switzerland, we consider IFS line 34ZF. Finally, for the United States, we take the corresponding series, i.e. either M1, IFS line 59MA or M1, OECD MEI. All seasonally unadjusted series were adjusted using monthly dummies.

- Industrial production: $\Delta\left(y_{t}-y_{t}^{U S}\right)$, where $y_{t}=\ln Y_{t}$ and $Y_{t}$ is the industrial production index, taken from IFS, line 66CZF, except for Switzerland for which no monthly series is available. For this country, we compute monthly observations from quarterly data (IFS, line 66) using the same procedure as in Molodstova and Papell (2009).

- Unemployment rate: $\Delta\left(u_{t}-u_{t}^{U S}\right)$, where $u_{t}=\ln U_{t}$ and $U_{t}$ is the unemployment rate from OECD MEI except for Germany / Euro area. For this 
country, we take a series from Datastream (Mnemonic WGUN\%TOTQ) that covers only West Germany and is thus unaffected by the German reunification that took place in 1990.

- Interest rate: $i_{t-1}-i_{t-1}^{U S}$, where $i_{t}$ is the monthly return calculated from the money market rate, IFS line 60B.

- Oil price: $\Delta p_{t}^{o i l}$, where $p_{t}^{o i l}=\ln P_{t}^{o i l}$ and $P_{t}^{o i l}$ is the average crude oil spot price from IFS. 


\section{References}

[1] Bacchetta, Philippe, and Eric van Wincoop (2004), "A Scapegoat Model of Exchange Rate Determination," American Economic Review, Papers and Proceedings 94, 114-118.

[2] Bacchetta, Philippe and Eric van Wincoop (2006), "Can Information Heterogeneity Explain the Exchange Rate Determination Puzzle?" American Economic Review 96, 552-576.

[3] Bacchetta, Philippe and Eric van Wincoop (2009), "Infrequent Portfolio Decisions: A Solution to the Forward Discount Puzzle," American Economic Review, forthcoming.

[4] Bacchetta, Philippe, Eric van Wincoop, and Toni Beutler (2009), "Can Parameter Instability Explain the Meese-Rogoff puzzle?" mimeo.

[5] Boivin, Jean (2006), "Has U.S. Monetary Policy Changed? Evidence from Drifting Coefficient and Real Time Data," Journal of Money, Credit and Banking 38(5).

[6] Canova, Fabio (2005), "Monetary Policy and the Evolution of the U.S. Economy," working paper, CREI.

[7] Cheung, Yin-Wong and Menzie David Chinn (2001), "Currency Traders and Exchange Rate Dynamics: A Survey of the US Market," Journal of International Money and Finance 20(4), 439-71.

[8] Cheung, Yin-Wong, Menzie David Chinn, and Antonio Garcia Pascual (2005), "Empirical Exchange Rate Models of the Nineties: Are Any Fit to Survive?" Journal of International Money and Finance 24, 1150-1175.

[9] Clarida, Richard, Jordi Gali and Mark Gertler(2000), "Monetary Policy Rules and Macroeconomic Stability: Evidence and Some Theory," Quarterly Journal of Economics 115(1), 147-180.

[10] Clark, Todd E. and Kenneth D. West (2006), "Using Out-of-sample Mean Square Prediction Errors to Test the Martingale Difference Hypothesis," Journal of Econometrics 135, 155-186. 
[11] Cogley, Timothy (2005), "Changing Beliefs and the Term Structure of Interest Rates: Cross-Equation Restrictions with Drifting Parameters," Review of Economic Dynamics 8, 420-451.

[12] Cogley, Timothy and Thomas J. Sargent (2005), "Drifts and Volatilities: Monetary Policies and Outcomes in the Post WWII U.S. ," Review of Economic Dynamics 82(2), 262-302.

[13] Del Negro, Marco and Christopher Otrok (2007), "Dynamic Factor Models with Time-Varying Parameters," mimeo.

[14] Elliott, Graham and Allan Timmermann (2008), "Economic Forecasting," Journal of Economic Literature 46, 3-56.

[15] Engel, Charles and Kenneth D. West (2005), "Exchange Rates and Fundamentals," Journal of Political Economy 113, 485-517.

[16] Fernandez-Villaverde, Jesus and Juan F. Rubio-Ramirez (2007), "How Structural are Structural Parameters?" working paper, Duke University.

[17] Hansen, L. and T. Sargent (2008), Robustness, Princeton University Press, forthcoming.

[18] Inoue, A. and B. Rossi (2007), "Which Structural Parameters Are "Structural"? Identifying the Sources of Instabilities in Structural Models," mimeo.

[19] Levin, A., A. Onatski, J. Williams, and N. Williams (2006), "Monetary Policy under Uncertainty in Micro-Founded Macroeconometric Models" in M. Gertler and K. Rogoff, eds., NBER Macroeconomics Annual 2005. Cambridge, MA: MIT Press.

[20] Meese, Richard A. and Kenneth Rogoff (1983a), "Empirical Exchange Rate Models of the Seventies: Do They Fit Out of Sample?" Journal of International Economics 14, 345-373.

[21] Meese, Richard A. and Kenneth Rogoff (1983b), "The Out-of-Sample Failure of Empirical Exchange Rate Models: Sampling Error or Misspecification?" in J. Frenkel (ed.), Exchange Rates and International Macroeconomics, 67-105, Chicago: University of Chicago Press. 
[22] Meese, Richard A. and Kenneth Rogoff (1988), "Was it Real? The Exchange Rate-Interest Differential Relation Over the Modern Floating-Rate Period," Journal of Finance 43, 933-948.

[23] Molodstova, Tanya and David Papell (2008), "Out-of-Sample Exchange Rate Predictability with Taylor Rule Fundamentals," mimeo.

[24] Nason, James N. and John N. Rogers (2008), "Exchange Rates and Fundamentals: A Generalization," International Finance Discussion Paper No. 948.

[25] Onatski, A. and N. Williams (2003), "Modeling Model Uncertainty," Journal of the European Economic Association 1, 1087-1022.

[26] Pastor, Lubos and Pietro Veronesi (2009), "Learning in Financial Markets," NBER WP 14646.

[27] Piazzesi, Monika and Martin Schneider (2007), "Equilibrium Yield Curves," in Daron Acemoglu, Kenneth Rogoff, and Michael Woodford (eds.), NBER Macroeconomics Annual 2006, MIT Press.

[28] Primiceri, Giorgio E. (2005), "Time Varying Structural Vector Autoregressions and Monetary Policy," Review of Economic Studies 72, 821-852.

[29] Rogoff, Rogoff and Vania Stavrakeva (2008), "The Continuing Puzzle of Short Horizon Exchange Rate Forecasting," NBER WP 14071.

[30] Rossi, Barbara (2006), "Are Exchange Rates Really Random Walks? Some Evidence Robust to Parameter Instability," Macroeconomic Dynamics 10, 2038.

[31] Sarno, Lucio and Giorgio Valente (2008), "Exchange Rates and Fundamentals: Footloose or Evolving Relationship?, " forthcoming Journal of the European Economic Association.

[32] Schinasi, Garry J. and P.A.V.B Swamy (1989), "The Out-of-Sample Forecasting Performance of Exchange Rate Models when Coefficients Are Allowed to Change," Journal of International Money and Finance 8, 375-390. 
[33] Sims, Christopher A. and Tao Zha (2006), "Were There Regime Switches in U.S. Monetary Policy?," American Economic Review 96(54-81).

[34] Stock, James, H. and Mark W. Watson (1996), "Evidence on Structural Instability in Macroeconomic Time Series Relations," Journal of Business and Economic Statistics 14, 11-30.

[35] Townsend, Robert M. (1983), "Forecasting the Forecasts of Others," Journal of Political Economy 91, 546-588.

[36] Wolff, Christian C.P. (1987), "Time-Varying Parameters and the Out-ofSample Forecasting Performance of Structural Exchange Rate Models," Journal of Business and Economic Statistics 5, 87-97. 


\section{Table 1 Benchmark Parameter Assumptions*}

\begin{tabular}{|c|c|}
\hline$T$ & 1000 \\
\hline$N$ & 5 \\
\hline$\beta$ & 1 \\
\hline$\sigma_{\beta}$ & 0.0165 \\
\hline$\sigma_{b}$ & 2.2 \\
\hline$\rho_{b}$ & 0.96 \\
\hline$\sigma_{v}$ & 2.7 \\
\hline$\phi_{1}$ & 0.06 \\
\hline$\phi_{2}$ & 0.1 \\
\hline$\sigma_{f}$ & 0.125 \\
\hline$\rho_{f}$ & 0 \\
\hline$\alpha$ & 33.3 \\
\hline
\end{tabular}

* Standard deviations are given in $\%$. 


\section{Table 2 Moments: Data and Model*}

\begin{tabular}{|l|c|c|c|c|}
\hline & Data & $\boldsymbol{\sigma}_{\boldsymbol{\beta}}=\mathbf{0}$ & $\begin{array}{r}\text { Benchmark } \\
\boldsymbol{\sigma}_{\boldsymbol{\beta}}=\mathbf{0 . 0 1 6 5}\end{array}$ & $\boldsymbol{\sigma}_{\boldsymbol{\beta}}=\mathbf{0 . 0 3 3}$ \\
\hline Standard Deviation $\Delta \mathrm{s}_{\mathrm{t}}$ & 2.91 & 2.90 & 2.99 & 3.04 \\
\hline $\operatorname{Corr}\left(\Delta \mathrm{s}_{\mathrm{t}}, \Delta \mathrm{s}_{\mathrm{t}-1}\right)$ & 0.04 & 0.04 & 0.04 & 0.04 \\
\hline Standard Deviation $\mathrm{i}_{\mathrm{t}}-\mathrm{i}_{\mathrm{t}}^{*}$ & 0.22 & 0.23 & 0.23 & 0.23 \\
\hline $\operatorname{Corr}\left(\mathrm{i}_{\mathrm{t}}-\mathrm{i}_{\mathrm{t}}^{*}, \mathrm{i}_{\mathrm{t}-1} \mathrm{i}_{\mathrm{t}-1}^{*}\right)$ & 0.92 & 0.92 & 0.93 & 0.93 \\
\hline $\operatorname{cov}\left(\Delta \mathrm{s}_{\mathrm{t}}, \mathrm{i}_{\mathrm{t}-1}-\mathrm{i}_{\mathrm{t}-1}^{*}\right) / \operatorname{var}\left(\mathrm{i}_{\mathrm{t}-1}-\mathrm{i}_{\mathrm{t}-1}^{*}\right)$ & -1.25 & -1.82 & -1.86 & -1.83 \\
\hline $\mathrm{R}^{2}$ monthly & 0.023 & 0.022 & 0.022 & 0.031 \\
\hline s.d. Monthly Change $\partial \Delta \mathrm{s}_{\mathrm{t}} / \partial \Delta \mathrm{f}_{\mathrm{nt}}$ & - & 0 & 25.9 & 45.0 \\
\hline s.d. Monthly Change $\Delta \beta_{\mathrm{nt}}$ & - & 0 & 0.30 & 0.61 \\
\hline
\end{tabular}

* Standard deviations are given in \%. 


\section{Table 3 Scapegoat Ratio*}

\begin{tabular}{|l|c|c|}
\hline & $\frac{s . d .\left(\tilde{\beta}_{n t}\right)}{\text { s.d. }\left(\Delta \beta_{n t}\right)}$ & $\begin{array}{c}\text { Scapegoat } \\
\text { ratio }\end{array}$ \\
\hline Benchmark Process & 319 & 86.3 \\
\hline Process 1 & 637 & 267.7 \\
\hline Process 2 & 7 & 1.6 \\
\hline Process 3 & 22 & 4.4 \\
\hline Process 4 & 49 & 10.1 \\
\hline
\end{tabular}

* The scapegoat ratio is the standard deviation of monthly changes in the reduced form derivative $\partial \Delta s_{t} / \partial \Delta \mathbf{f}_{n t}$ of the exchange rate with respect to fundamentals relative to the standard deviation of monthly changes in structural parameters. 


\section{Figure 1 Derivative $\Delta \mathrm{s}_{\mathrm{t}}$ with respect to $\Delta \mathrm{f}_{\mathrm{nt}}(10 \text { years })^{*}$}
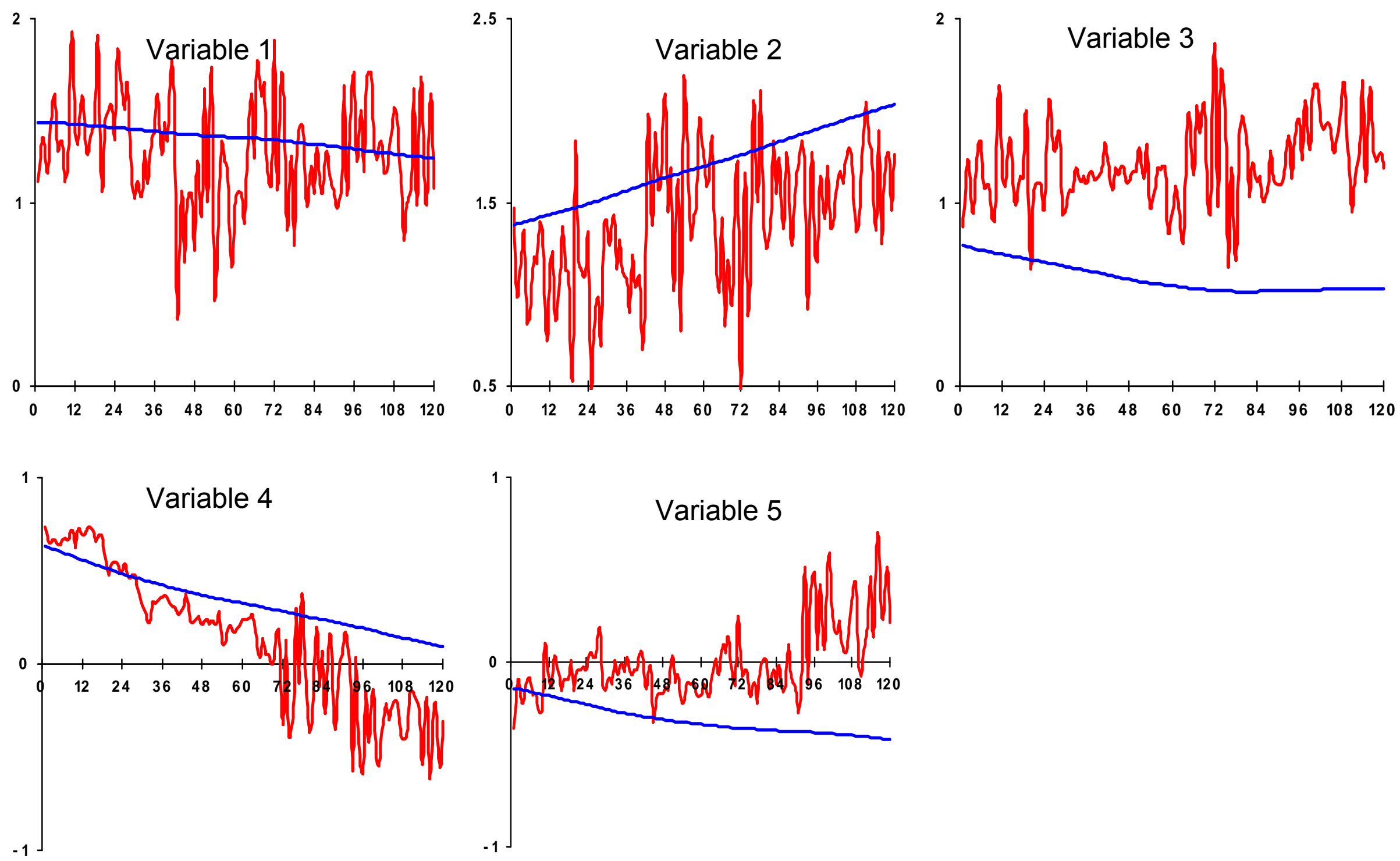

* The smooth line is $\beta_{n t}$, while the volatile line represents the derivative of $\Delta \mathrm{s}_{\mathrm{t}}$ with respect to $\Delta \mathrm{f}_{\mathrm{nt}}$. 


\section{Figure 2 Derivative $\Delta s_{t}$ with respect to $\Delta f_{n t}(100 \text { years })^{*}$}
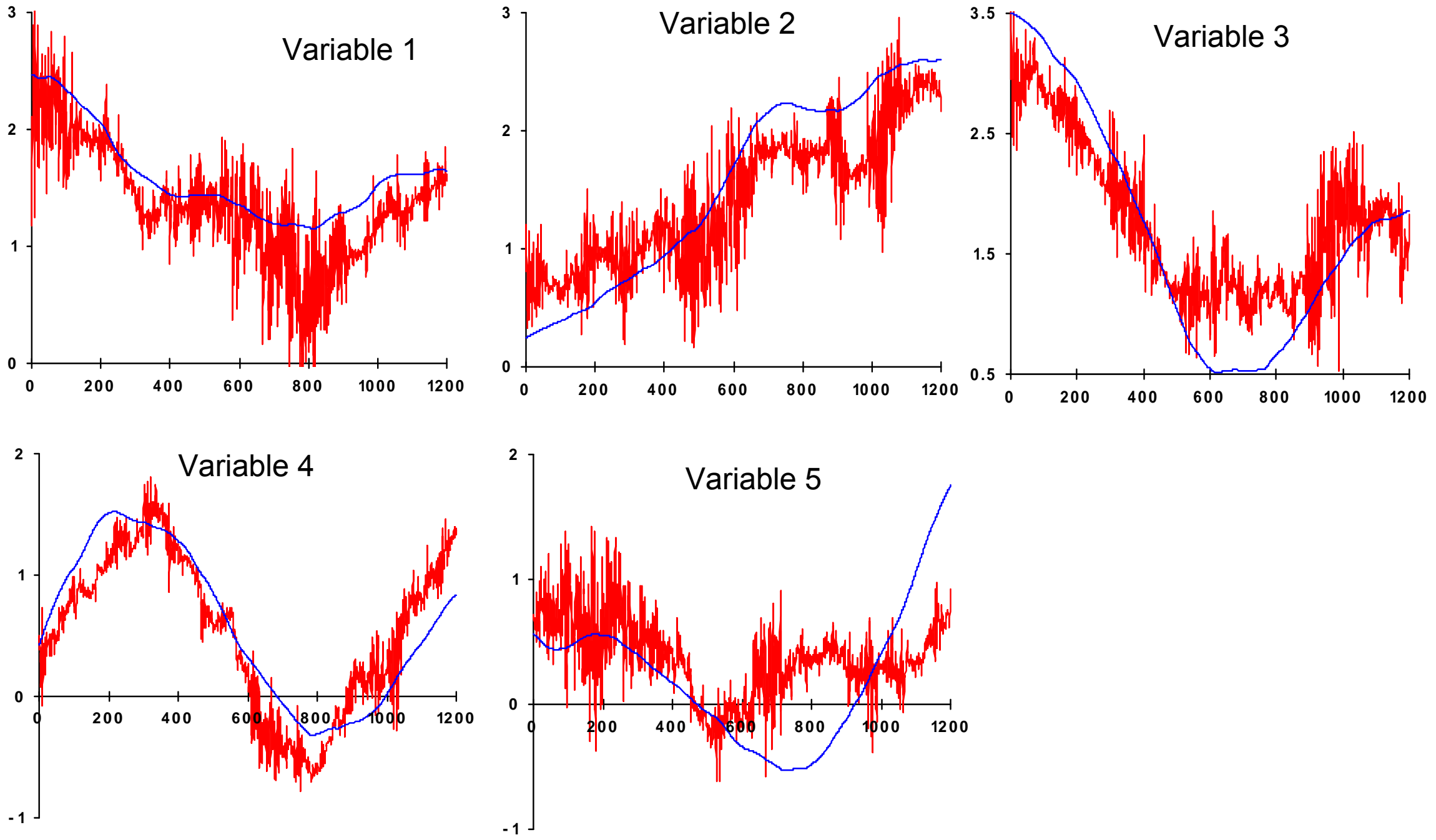

\footnotetext{
* The smooth line is $\beta_{n t}$, while the volatile line represents the derivative of $\Delta \mathrm{s}_{\mathrm{t}}$ with respect to $\Delta \mathrm{f}_{\mathrm{nt}}$.
} 
Figure 3 Expectations $\beta_{n t}$ (variable 1)
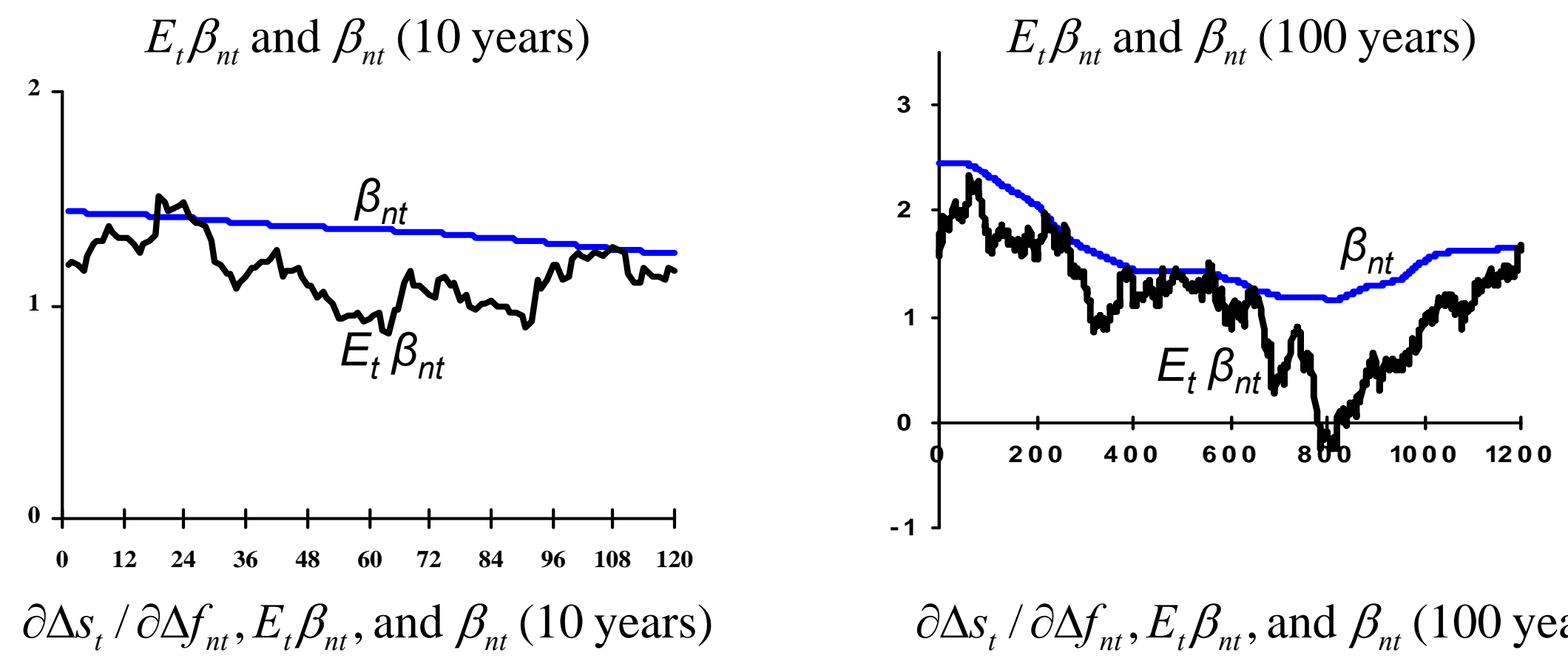

$$
\partial \Delta s_{t} / \partial \Delta f_{n t}, E_{t} \beta_{n t} \text {, and } \beta_{n t} \text { (100 years) }
$$
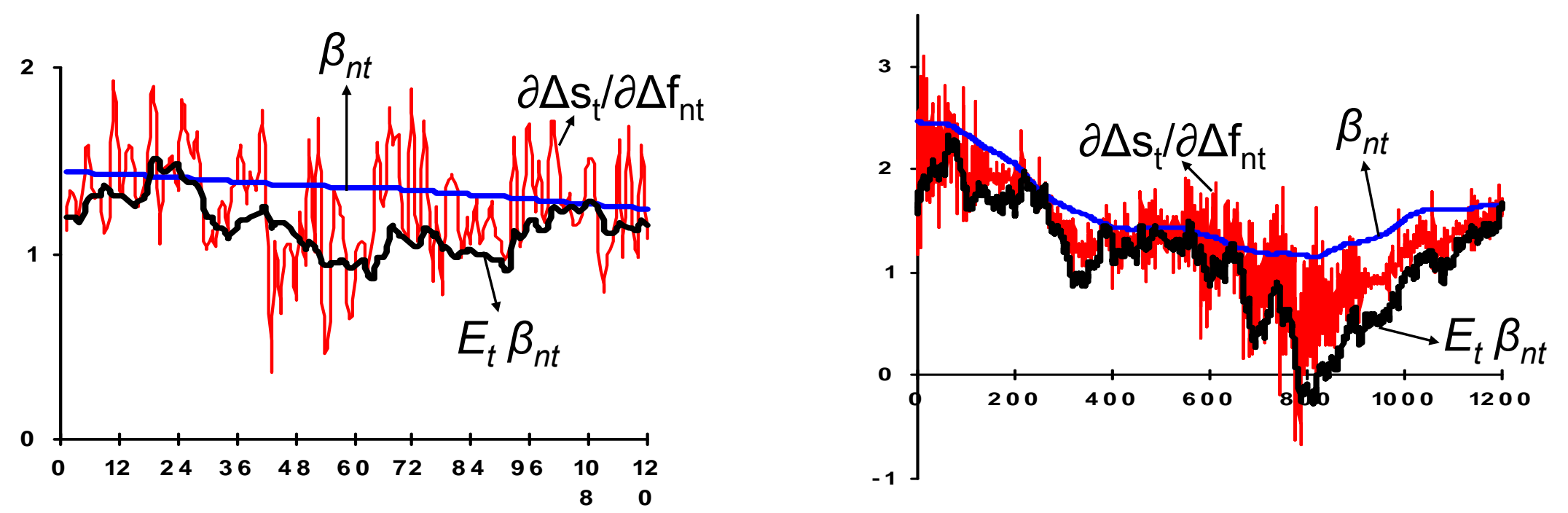


\section{Figure 4 Impulse Response Functions for Alternative Processes for Structural Parameters*}
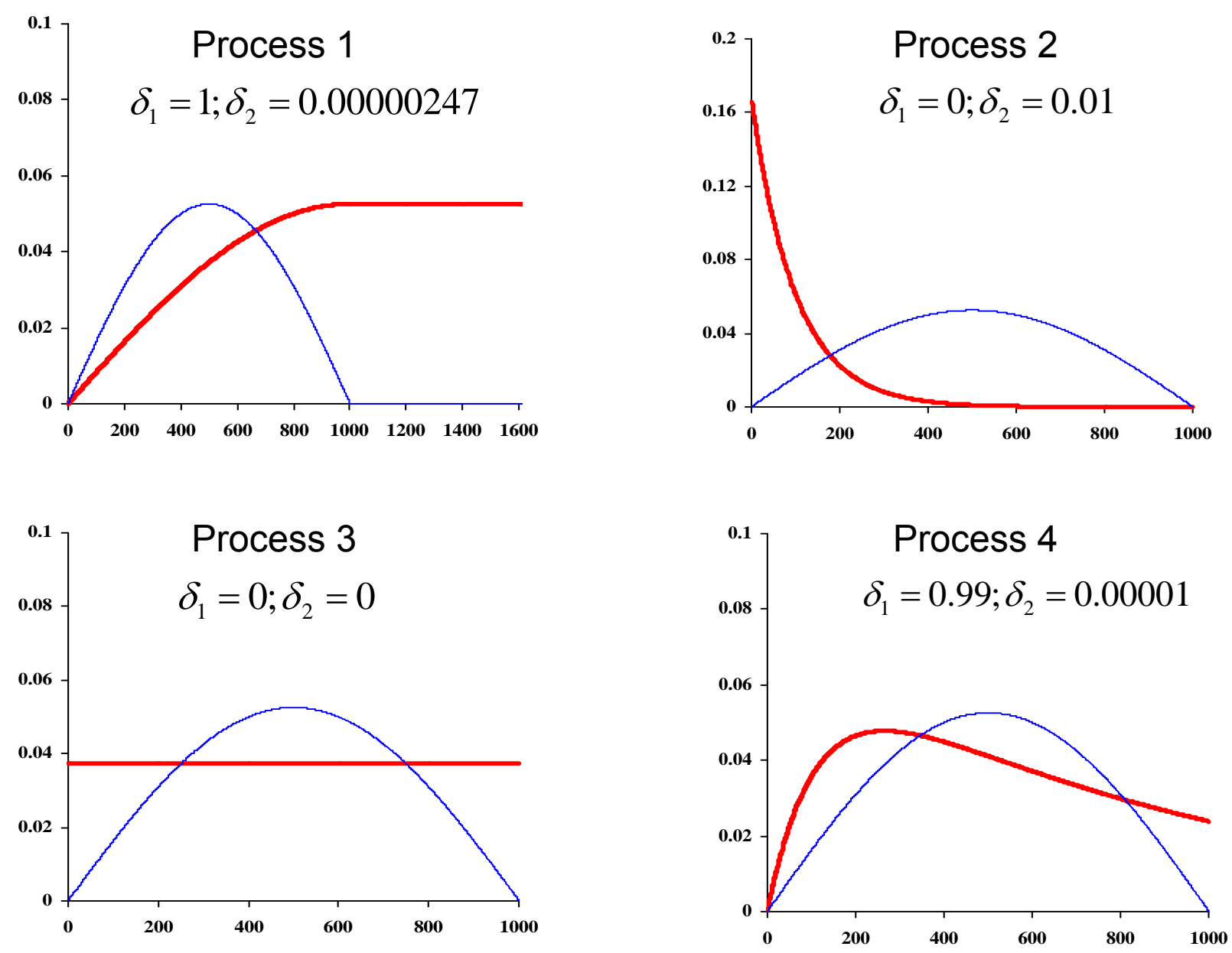

* Each graph shows the impulse response functions of the structural parameters in response to one standard deviation parameter innovations. The different charts correspond to different values for $\delta_{1}$ and $\delta_{2}$ for the process described in the paper. For comparison each graph also shows the impulse response function for the benchmark process (thinner humpshaped line). 
Figure 5 Derivative $\Delta \mathrm{s}_{\mathrm{t}}$ with respect to $\Delta \mathrm{f}_{\mathrm{nt}}(100 \text { years })^{*}$ Process 1
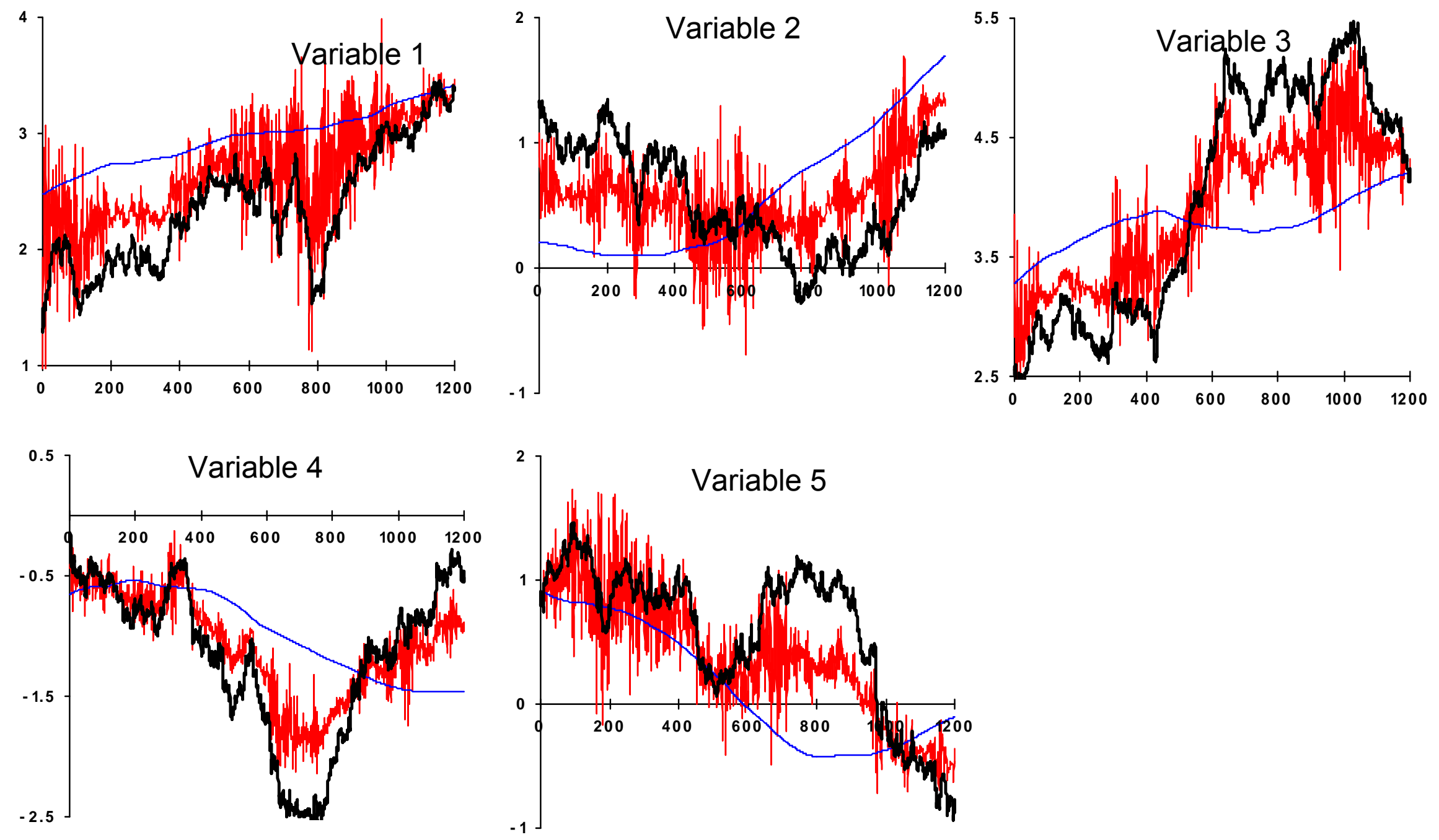

* The smooth thin line is $\beta_{n t}$ the thick line is $\mathrm{E}_{t} \beta_{n t}$, while the most volatile line represents the derivative of $\Delta \mathrm{s}_{\mathrm{t}}$ with respect to $\Delta \mathrm{f}_{\mathrm{nt}}$. 


\section{Figure 6 Unobservable Shocks and Scapegoat Ratio*}

\section{Scapegoat Ratio}

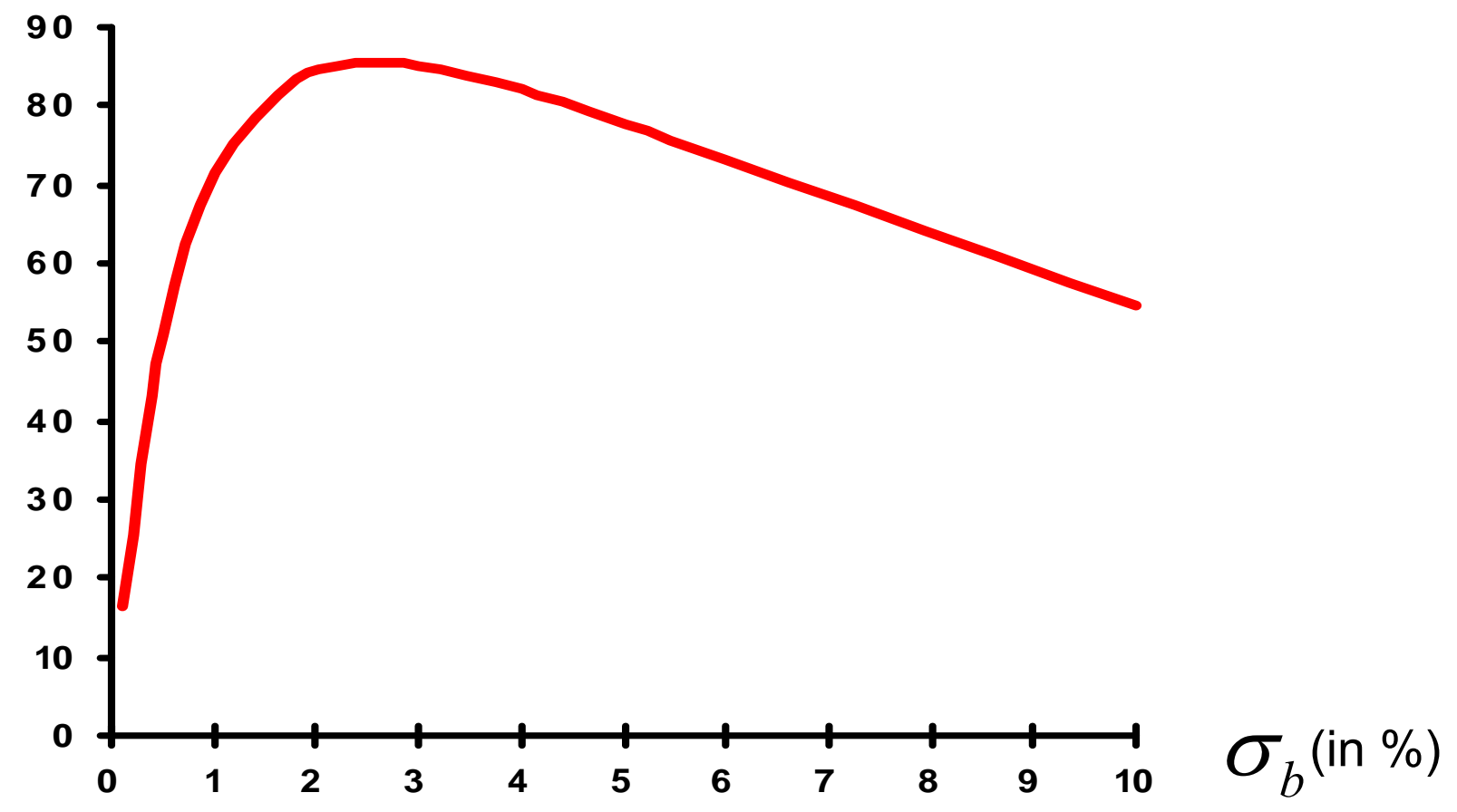

*Scapegoat ratio=standard deviation monthly changes in $\partial \Delta s_{t} / \partial \Delta f_{n t}$ relative to standard deviation monthly changes in $\beta_{n t}$. 


\section{Figure 7 Out-of-Sample Fit and Sample Size Empirical Evidence}

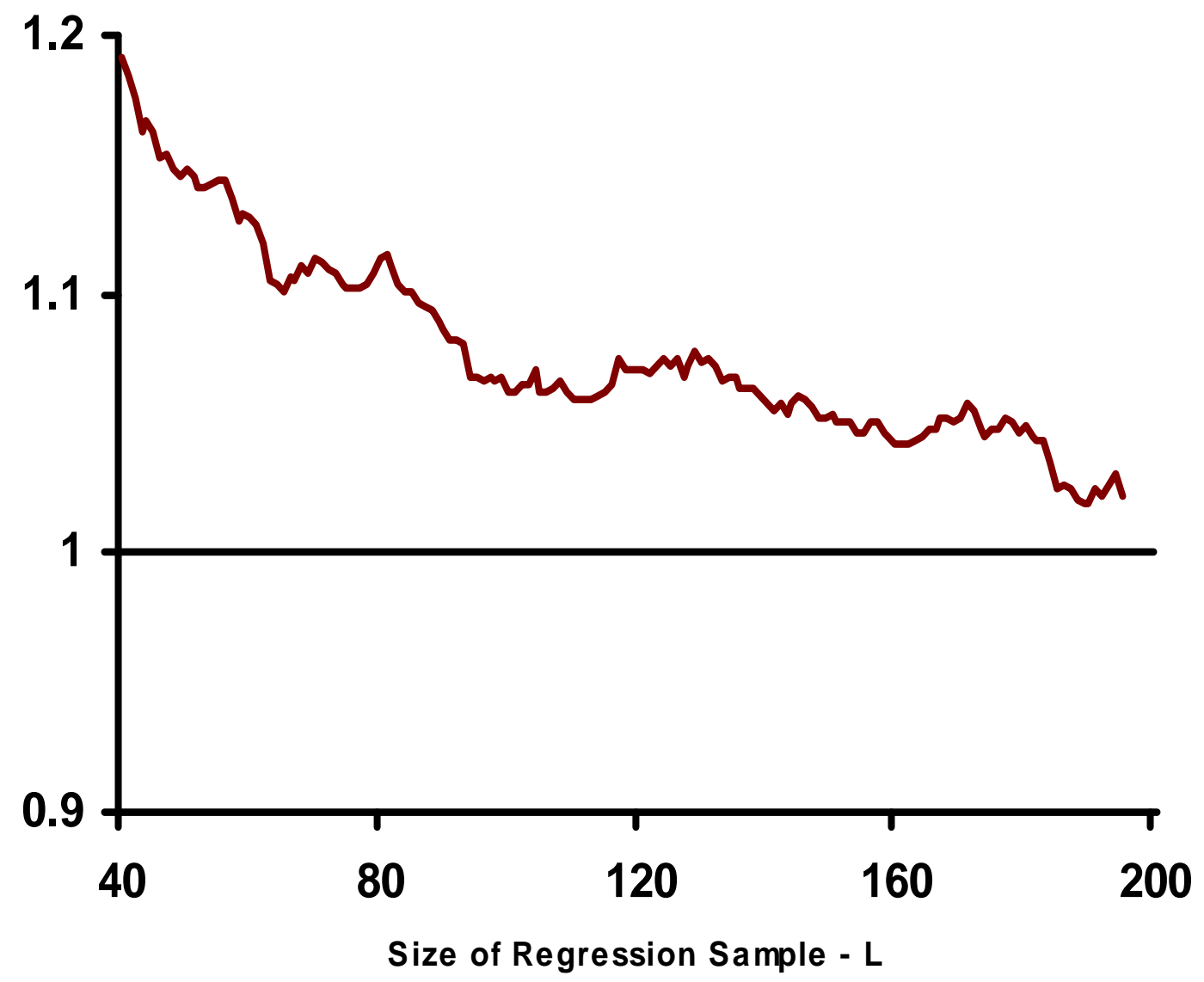

Note: Mean-Square Error (MSE) of one-month ahead exchange rate forecasts from model including changes in money, output, unemployment rate, oil price and level of lagged interest rate estimated by rolling regressions relative to MSE of random walk forecast. The reported line is an average for bilateral US dollar exchange rate with Canadian dollar, Japanese yen, Swiss franc, British pound and German mark - Euro composite. Forecasting sample is 200 periods. Sample : 1975M9 - 2008M9. Data sources: IFS and OECD. 


\section{Figure 8 Out-of-Sample Forecasting: MSE Model/MSE Random Walk}

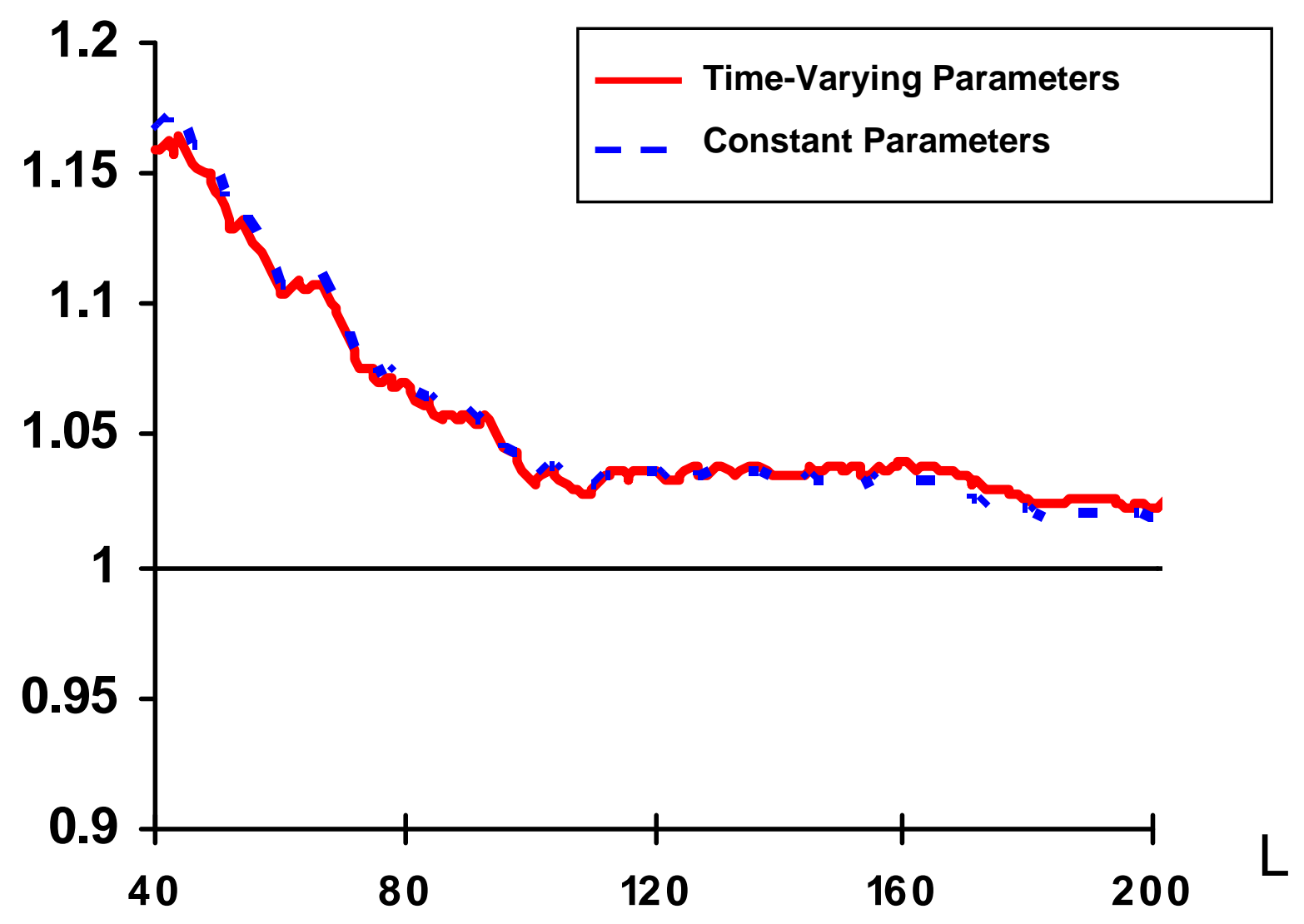

